\title{
Alzheimer's Disease Prevention and Treatment: Case for Optimism
}

\author{
Vladimir Volloch ${ }^{1^{*}}$, Bjorn Olsen ${ }^{1}$ and Sophia Rits ${ }^{2,3}$ \\ 'Department of Developmental Biology, Harvard School of Dental Medicine, USA \\ ${ }^{2}$ Division of Molecular Medicine, Children's Hospital, Boston, USA \\ ${ }^{3}$ Department of Biological Chemistry and Molecular Pharmacology, Harvard Medical School, USA
}

\begin{abstract}
A paradigm shift is under way in the Alzheimer's field. A view of Alzheimer's disease, AD, prevailing until now, the old paradigm, maintains that it is initiated and driven by the overproduction and extracellular accumulation of beta-amyloid, $A \beta$; a peptide assumed to be derived, both in health and disease, solely by proteolysis of its large precursor, $\beta$ APP. In $\mathrm{AD}$, according to this view, $\mathrm{A} \beta$ overproduction-associated neurodegeneration begins early, accumulates throughout the lifespan, and manifests symptomatically late in life. A number of drugs, designed within the framework of exceptionality of the $\beta$ APP proteolytic/ secretory pathway in $\mathrm{A} \beta$ production in Alzheimer's disease, achieved spectacular successes in treatment, even the reversal, of $A D$ symptoms in animal models. Without exception, they all exhibited equally spectacular failures in human clinical trials. This paradigm provides few causes for optimism with regard to prevention and treatment of $\mathrm{AD}$. In its context, the disease is considered untreatable in the symptomatic phase; even prodromal cases are assumed too advanced for treatment because $A \beta$-triggered damages have been accumulating for preceding decades, presumably starting in the early twenties and, to be effective, this is when therapeutic intervention should commence and continue for life. The new paradigm does not dispute the seminal role of $A \beta$ in $A D$ but posits that beta-amyloid produced in the $\beta$ APP proteolytic/secretory pathway causes $\mathrm{AD}$ in humans no more than it does in non-human mammals that share this pathway with humans, accumulate $A \beta$ as they age, but do not develop the disease. Alzheimer's disease, according to this outlook, is driven by the $\mathrm{AD}$-specific pathway of $\mathrm{A} \beta$ production, independent of $\beta \mathrm{APP}$ and absent in animals. Its activation, late in life, occurs through accumulation, via both cellular uptake of secreted $A \beta$ and neuronal retention of a fraction of beta-amyloid produced in the $\beta$ APP proteolytic pathway, of intraneuronal A $\beta$, which triggers mitochondrial dysfunction. Cellular stresses associated with mitochondrial dysfunction, or, probably, the integrated stress response, ISR, elicited by it, activate an $\mathrm{AD}$-specific $\mathrm{A} \beta$ production pathway. In it, every conventionally produced $\beta A P P$ mRNA molecule potentially serves repeatedly as a template for production of severely 5'-truncated mRNA encoding C99 fragment of $\beta A P P$, the immediate precursor of $A \beta$ that is processed in a non-secretory pathway, apparently in a neuron-specific manner. The resulting intraneuronally retained $A \beta$ augments mitochondrial dysfunction, which, in turn, sustains the activity of the $\beta A P P$ mRNA amplification pathway. These self-propagating $A \beta$ overproduction/mitochondrial dysfunction mutual feedback cycles constitute the engine that drives $\mathrm{AD}$ and ultimately triggers neuronal death. In this paradigm, preventive treatment can be initiated any time prior to commencement of $\beta A P P$ mRNA amplification. Moreover, there are good reasons to believe that with a drug blocking the amplification pathway, it would be possible not only to preempt the disease but also stop and reverse it even when early AD symptoms are already manifested. Thus, the new paradigm introduces a novel theory of Alzheimer's disease. It explains the observed discordances, determines defined therapeutic targets, provides blueprints for a new generation of conceptually distinct AD models and specifies design of a reporter for the mRNA amplification pathway. Most importantly, it offers detailed guidance and tangible hope for prevention of the disease and its treatment at the early symptomatic stages.
\end{abstract}

Keywords: $\beta$ APP-independent generation of $A \beta$; Asymmetric RNA-dependent $\beta$ APP mRNA amplification; Intraneuronal retention of A $\beta$; AD models for the new paradigm; Universal reporter for the mammalian RNA-dependent mRNA amplification process; Reporter-based optimal AD models

Old Paradigm: Alzheimer's Disorder is a "Slow" Disease Commencing Early in Life and Untreatable in the Symptomatic Phase; To be Effective, Preventive Therapy Should Start Early On and Continue for Life

The currently consented paradigm provides few causes for optimism with regard to prevention and treatment of Alzheimer's

Citation: Volloch V, Olsen B, Rits S. Alzheimer's Disease Prevention and Treatment: Case for Optimism. Ann Integr Mol Med. 2020; 2(1): 1008.

Copyright: @ 2020 Vladimir Volloch

Publisher Name: Medtext Publications LLC

Manuscript compiled: Aug 12 $2^{\text {th }}, 2020$

*Corresponding author: Vladimir Volloch, Department of Developmental Biology, Harvard School of Dental Medicine, USA, E-mail: vladimir.volloch@gmail.com; vladimir_volloch@hms.harvard.edu disease, $\mathrm{AD}$. In this paradigm, it is strongly believed that $\mathrm{AD}$ is initiated and driven by overproduction and extracellular accumulation of betaamyloid, $A \beta$. This peptide, it is assumed, is derived, both in health and disease, solely by two proteolytic cleavages of a large beta-amyloid precursor protein, $\beta$ APP, with beta-secretase (beta-site amyloid precursor protein cleaving enzyme, BACE) cleavage between residues 671 and 672 generating the C-terminal fragment of $\beta A P P, C 99$, and forming the $\mathrm{N}$-terminus of $\mathrm{A} \beta$, and subsequent gamma-secretase (gamma-site $\beta$ APP cleaving enzyme) cleavage of C99, forming the $\mathrm{C}$-terminus of $\mathrm{A} \beta$ and thus completing its production. In $\mathrm{AD}$, according to this view, $A \beta$ overproduction and associated damages begin early, accumulate slowly throughout the lifespan, and manifest symptomatically late in life in sporadic cases, SAD, or around the fifties in familial cases, FAD. These notions were formalized in the Amyloid Cascade Hypothesis, ACH, which became a prevailing evidence-based theory of $\mathrm{AD}$, and $\mathrm{A} \beta$ emerged as the most compelling therapeutic target of the disease. In recent years, a large number of potential AD drugs, targeting either the $\beta A P P$ proteolytic pathway or the extracellular $A \beta$ directly, have been developed. They include inhibitors of beta-secretase such as atabecestat, lanabecestat, 
AZD3839, LY2811376, LY2886721, verubecestat; inhibitors and modulators of gamma-secretase such as avagacestat, begacestat, semagacestat, tarenflurbil; inhibitors of beta-amyloid aggregation such as scillo-inositol, PBT2, tramiprosate; A $\beta$-based antigens such as vanutide, AD02, CAD-106, AN-1792; and both polyclonal and monoclonal $A \beta$-targeting antibodies such as crenezumab, gantenerumab, solanezumab, bapineuzumab and ponezumab. Many of these potential drugs achieved spectacular successes in treatment, even reversal, of $\mathrm{AD}$ symptoms in animal models. They all, however, exhibited equally spectacular failures in human clinical trials. To explain this discordance, it was suggested that the drugs were administered too late in the course of the disease in human trials Therefore, the trials, initially involving mild-to-moderate $\mathrm{AD}$ patients, were repeated with patients suffering from mild cognitive impairment and biomarker evidence of $\mathrm{A} \beta$ deposition in brain (prodromal $\mathrm{AD}$ ). The outcomes, however, did not change; moreover, in a number of trials the administration of drugs actually worsened the cognitive or clinical condition of the subjects. The currently prevailing explanation is that even the prodromal cases are way too advanced for treatment because $\mathrm{A} \beta$-triggered damages have been accumulating for decades, presumably starting in the early twenties, and, to be effective, this is when therapeutic intervention should commence and continue the entire life.

New Paradigm: Alzheimer's Disorder is a "Fast" Disease Commencing Late in Life, Preventable by Therapeutic Intervention Initiated Any Time Prior to the Symptomatic Phase and Treatable at the Early Symptomatic Stages

"How often have I said to you that when you have eliminated the impossible, whatever remains, however improbable, must be the truth?" Sherlock Holmes in The Sign of the Four [1].

\section{Indications for the Occurrence of Intraneuronally Retained $A \beta$ Produced in AD-Specific Pathway Independently of $\beta A P P$}

The new paradigm, suggested in studies by Volloch and coinvestigators [2-8], does not dispute the seminal role of $A \beta$ in triggering and sustaining the disease. It introduces, however, a distinct notion and an entirely different interpretation of the $\mathrm{AD}$-specific source of $A \beta$ overproduction and its mode of operation in the disease. To understand the discordance between the outcomes seen in animal models and those obtained in human clinical trials, it is instructive to address the apparent exclusivity of the disease to Homo sapiens. All non-human mammals tested to date accumulate $A \beta$ as they age but they do not develop $A D$. This is true for small-sized, short-lived animals such as mice, and for large-sized, long-lived non-human mammals such as elephants. In contrast, humans do both. It appears, therefore, that the extent of increase in beta-amyloid levels, sufficient to trigger a cascade of molecular events culminating in $\mathrm{AD}$, can be reached physiologically in Alzheimer's disease but not in non-human mammals or in healthy humans. This disparity could be easily explained if different pathways of beta-amyloid generation were involved in these instances. It is clear, however, that the same $\mathrm{A} \beta$ production pathway, namely proteolysis of beta-amyloid precursor protein, is shared by non-human mammals, healthy humans and $\mathrm{AD}$ patients. It can be suggested, therefore, that in $\mathrm{AD}$, in addition to the common beta-amyloid production pathway, another, qualitatively different pathway of A $\beta$ generation, exclusive to Alzheimer's disease, is in operation, and it is this pathway that drives the disease [6-8]. Two key features of this pathway can be deduced from the results of multiple human clinical trials. First, since BACE inhibitors, highly efficient in animal models, have no therapeutic effect whatever in $\mathrm{AD}$ patients, it appears that the $A D$-specific $A \beta$ production pathway is both $\beta A P P$-independent and the predominant process of beta-amyloid generation in Alzheimer's disease [6-8]. Second, since human trials of treatments directly targeting extracellular $\mathrm{A} \beta$, i.e. beta-amyloid immunotherapy, decidedly successful in animal models, and its significant reduction by BACE inhibitor, indicative that the inhibitor reached neurons and blocked $\beta \mathrm{APP}$ proteolysis! [9], are also completely ineffective in Alzheimer's disease, beta-amyloid produced in the predominant, $A D$-specific, $\beta A P P$-independent pathway appears to be retained intraneuronally $[7,8]$. These conclusions accentuate the inadequacy of current animal models of $\mathrm{AD}$, all based on a notion of the exceptionality of the $\beta \mathrm{APP}$ proteolytic/secretory pathway in $\mathrm{A} \beta$ production in Alzheimer's disease, in assessing the impact of potential AD drugs. They also necessitate a new generation of conceptually distinct $\mathrm{AD}$ models as well as comprehensive reassessment of our perception of the disease.

\section{AD-Specific, BAPP-independent Pathway of Beta-Amyloid Production}

Our understanding of Alzheimer's disease-specific pathway of $A \beta$ production described in recent studies [6-8] is suggested by the results obtained in systems overproducing specific proteins [10-13]. This pathway is driven by RNA-dependent amplification of specific mammalian mRNAs, a process diagrammed in the top and middle panels of Figure 1, which can be briefly summarized as follows. The amplification process occurs in the cytoplasm and starts with transcription of the antisense complement from a conventional, spliced mRNA template, initiating at the 3'poly(A), possibly with the help of a uridylated protein, as seen in viral RNA-dependent RNA synthesis, RdRs (Step 1), and terminating at the 3'end with the "C", a transcript of the capG of mRNA [11-13]. Generation of a complete antisense transcript requires the presence of an eligible RNA template and a compatible polymerase activity. The only major prerequisite for a potential RNA template appears to be the presence of the poly(A) segment at its 3 ' terminus $[10-12,14]$. The compatible enzymatic activity is RNA-dependent RNA polymerase, RdRp. The RdRp activity in mammalian cells appears to be non-conventional; two possible candidates for this role are the RNA polymerase II complex or its components [15,16] and RdRp activity of the TERT complex [17], both ubiquitously present in all cells. Under regular circumstances, the RdRp activity in mammalian cells produces only short antisense RNA transcripts [14]. It appears that the component responsible for the production of long antisense transcripts in mammalian cells is a processivity conferring co-factor of RdRp that is induced, possibly along with other inducible components of RdRp complex, under circumstances when overproduction of specific proteins is required, by different types of cellular stress, or, more likely, by the integrated stress response elicited by different types of cellular stress [11-13].

The resulting double stranded sense/antisense structure is separated into single-stranded molecules by a helicase activity that mounts the poly(A) segment of the 3'poly(A)-containing strand (the sense-oriented strand) of the double helical structure and proceeds along this strand modifying, on average, every fifth nucleotide in the process $[11,12]$ (Step 2). Only purines, the "As" and the "Gs" appear to be modified in the separation/modification process $[11,12]$. The 5 ' poly $(\mathrm{U})$-containing antisense strand remains unmodified during and after the separation [10-12]; this being essential, as described below, for the production of a new sense strand, since modifications were shown to interfere with, and would preclude, complementary interactions required in this process $[11,12]$. 


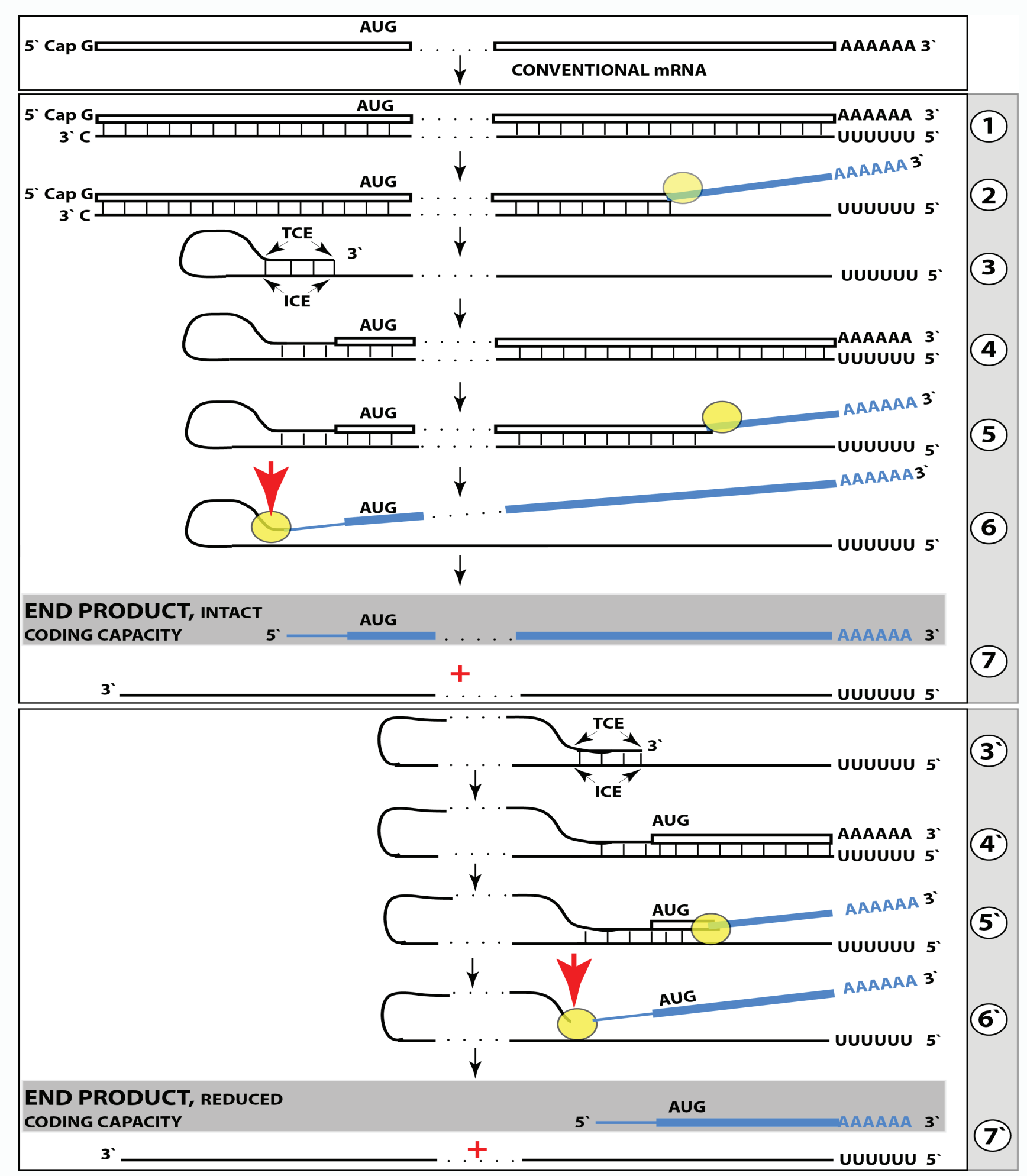

Figure 1: Projected stages of the chimeric pathway of RNA-dependent amplification of mammalian mRNA; the process can result in a 5'-truncated molecule encoding the C-terminal fragment of a conventionally encoded polypeptide. Boxed line-sense strand RNA. Single line-antisense strand RNA. "AUG"-functional translation initiation codon (could be other than AUG). "TCE"-3'-terminal complementary element; "ICE"- internal complementary element, both on the antisense RNA strand. Yellow circle - helicase/modifying activity complex. Blue lines (both single and boxed) - RNA strand modified and separated from its complement by a helicase complex. Red arrow - position of cleavage of the chimeric intermediate. Step 1: Synthesis of antisense strand; step 2: Strand separation; step 3: Folding of antisense strand into self-priming configuration; step 4: Extension of self-primed antisense RNA; step 5: Strand separation; step 6: Cleavage of the chimeric intermediate; step 7: End-products of RNA amplification. Steps 3'-7' correspond to steps 3-7. Top panel: Conventional, genome-transcribed mRNA molecule. Middle panel: Projected stages of RNA-dependent mRNA amplification. “ICE” is located within a segment of antisense RNA corresponding to the 5'UTR of conventional mRNA; the chimeric RNA end product contains the entire coding content of conventional mRNA. Bottom panel: "ICE" is located within a segment of antisense RNA corresponding to the coding region of conventional mRNA. The amplified chimeric end product contains a 5'-truncated coding region of conventional mRNA. The translational outcome is decided by position of the first functional translation initiation codon; if in-frame, a CTF of conventional polypeptide is produced. 
The specificity of mRNA amplification appears to be determined at the 3 ' terminus of an antisense transcript by its ability or inability to support production of a complementary sense strand RNA molecule, the end product of the amplification process. The generation of a sense strand on an antisense template occurs via the extension of the 3' terminus of a self-primed antisense template and requires the presence within the antisense transcript of two spatially independent, yet topologically compatible, i.e. mutually accessible within the folded RNA molecule, complementary elements [10-12,18]. One of these is the strictly 3'-Terminal Complementary Element (TCE), the other is the Internal Complementary Element (ICE). These elements (Step 3) must be complementary to a sufficient extent to form a priming structure but may contain mismatches and utilize unconventional G/U pairings [10-12]. In addition, the self-priming structure must accommodate the additional 3 '-terminal "C", a transcript of the 5'cap"G" of mRNA [11-13]. The generation of a sense strand requires the thermodynamic feasibility, enhanced/enabled by the occurrence of two complementary and topologically compatible elements, of the antisense strand folding into a self-priming configuration.

Provided that a self-priming structure is formed, the $3^{\prime}$ end of the folded antisense strand is extended by RdRp into a sense-orientation molecule terminating with the poly(A) at the 3'end (Step 4), thus generating a hairpin-structured chimeric intermediate consisting of covalently joined sense and antisense strands. The double stranded portion of the resulting structure is separated by a helicase activity invoked above, which mounts the 3'poly(A) of a newly synthesized sense strand component of the chimeric intermediate and proceeds along this strand in the $5^{\prime}$ direction, modifying the molecule as it advances (Step 5). When the helicase activity reaches a singlestranded portion of the hairpin structure, it, or associated activities, cleave the molecule either within the TCE, at a TCE/ICE mismatch, or immediately upstream of the TCE (red arrowhead, Step 6); the cleavage occurs between the 5' hydroxyl group and the 3' phosphate $[11,12]$.

Strand separation, in conjunction with the cleavage, produces two single-stranded molecules (Step 7) one of which is a chimeric mRNA, the functional mRNA end product of amplification and the basis for defining this pathway as the "chimeric". The chimeric nature of this end product is due to the presence at its 5' end of a 3 '-terminal segment of the antisense strand consisting, depending on the site of cleavage of the chimeric intermediate, of either the entire TCE or a portion thereof covalently attached, in a 5' to 3 ' orientation, to the $5^{\prime}$-truncated sense strand. This chimeric molecule is modified and 3' polyadenylated. In contrast to conventional mRNA that can be repeatedly used as RdRp template, it cannot be further amplified because its antisense complement would lack the TCE, but can be translated into the conventional mRNA-encoded polypeptide $[11,12]$ If, as suggested above, the RNA-dependent mRNA amplification pathway is indeed activated by the integrated stress response (ISR) apparatus, one of the plausible functions of nucleotide modifications of the amplified chimeric mRNA, in addition to those described in [11], could be rendering it compatible, in contrast to the bulk of conventionally produced mammalian mRNA species, with the phosphorylated translation initiation factor eIF $2 \alpha$, a feature of the the ISR. Interestingly, in addition to suppressing, through this feature, translation of the bulk of conventionally produced mRNA, the ISR enables expression/translation of select transcription factors $[19,20]$; some of these transcription factors could, in fact, be inducible components of the mammalian $m R N A$ amplification pathway postulated above or the enablers of their expression. Moreover, it follows that, in such a case, mRNA produced in the amplification pathway is not only exceptionally abundant $[11,12]$ due to the method of its generation, but is also preferentially translated. In the chimeric pathway of mRNA amplification, the cleavage of the chimeric intermediate, following the strand separation and the associated modification of the poly(A)containing strand of the double-stranded hairpin structure, is the ultimate act in the generation of the chimeric mRNA end product. Consequently, it is formed already modified and is never present in the unmodified form $[11,12]$. Therefore, because the modified amplified RNA is resistant to reverse transcription $[11,12]$, it cannot, in contrast to chimeric RNA intermediates, be detected by conventional reverse transcription-based sequencing methods.

In the scenario discussed above, both complementary elements required for an appropriate folding and self-priming of the antisense strand, the TCE and the ICE, are located within its segment corresponding to the 5'UTR of a conventional genome-encoded mRNA. In such a situation, the chimeric RNA end product contains the entire protein-coding region of a conventional mRNA and can be translated into the original, conventional mRNA-encoded, polypeptide. In the chimeric mRNA amplification pathway, the position of the TCE within the antisense molecule is fixed: It is always strictly 3'-terminal. In contrast, the intramolecular location of the internal ICE element is variable, and potentially it can be positioned within a segment of the antisense strand corresponding to the coding portion of an mRNA, a scenario diagrammed in steps 3' through 7' of the bottom panel of Figure 1. In this scenario, the chimeric RNA end product consists of a 3'-terminal segment of the antisense strand (the TCE or its fraction) attached, in a 5' to 3' orientation, to a 3' portion of a conventional mRNA progenitor with a 5 '-truncated coding region. In such a case, the translational outcome would be decided by the position of the first functional (capable of initiation of translation) AUG or another translation initiation-competent codon. If it were inframe with the protein-encoding information content of conventional mRNA, translation would result in the C-terminal fragment, CTF, of a conventionally encoded polypeptide. This variant of RNA-dependent mRNA amplification pathway would be asymmetric. Indeed, only one end, a 3'-terminal portion of the coding region (+UTR) of conventional mRNA, would be amplified, and its translation would potentially produce only one end of a conventional genome-encoded polypeptide, its C-terminal fragment. With regard to a possible $\beta A P P-i n d e p e n d e n t$ production of beta-amyloid in such a manner, three crucial questions are: (1) Is human $\beta A P P$ mRNA eligible for RNA-dependent amplification? (2) If it is, does amplification occur asymmetrically? (3) If affirmative, would the N-terminus of the resulting polypeptide be precisely that of $\mathrm{A} \beta$ ? (It can be longer than $A \beta$ at its $C$-end and be trimmed to size by gamma-secretase cleavage).

\section{Potentially Pivotal Role of the AUG Encoding Met671 in Conventional Human $\beta A P P$ mRNA in $\beta A P P-I n d e p e n d e n t$ Generation of $A \beta$ in Alzheimer's Disease}

The answer to question (3) above is suggested by the primary structure of the human $\beta A P P$ mRNA. In this molecule, the A $\beta$ encoding segment is preceded immediately and in-frame by the AUG codon encoding methionine in position 671 of the $\beta A P P$, the position of the BACE-mediated cleavage (671/672). If translation were initiated at this position, it would produce, after the removal of the $\mathrm{N}$-terminal methionine by the N-terminal methionine aminopeptidase, C99 fragment, the immediate precursor of $A \beta$, independently of $\beta A P P$. Interestingly, the AUG in question is situated within a nucleotide context optimal for the initiation of translation (an "A" in position -3 and a " $G$ " in position +4 relative to the " $A$ " of the AUG codon). In fact, of 
the twenty AUG codons encoding methionine residues in the human $\beta A P P$ mRNA, only the AUG encoding Met671 (not even the AUG encoding Met1) is located within an optimal translation initiation context. Such favorable positioning of the AUG encoding Met671 of $\beta$ APP was the basis for a proposal that in Alzheimer's disease, the C99 fragment of beta-amyloid precursor protein may be generated independently from $\beta$ APP by the internal initiation of translation at the AUG encoding Met671 in the intact $\beta$ APP mRNA [21]. Such precursor-independent generation of $\mathrm{C} 99$ would be an efficient way to overproduce $A \beta$. This is because (a) C99 is not susceptible to the alpha-secretase cleavage, and (b) cleavage by gamma-secretase was shown to be not the rate-limiting step in the production of $A \beta[22-$ 24]. The possibility of internal initiation of translation, proposed in [21], has been, however, subsequently ruled out by experiments of Citron and co-investigators [25].

On the other hand, the implications of the occurrence of an AUG codon in such a position for potential $\beta$ APP-independent generation of $A \beta$ via asymmetric $\beta A P P$ mRNA amplification are clear: If human $\beta A P P$ mRNA is eligible for RNA-dependent amplification, if it is amplified in an asymmetric manner in $\mathrm{AD}$, and if, in the resulting 5'-truncated chimeric mRNA, the first, 5'-most, functional translation initiation codon were the AUG encoding Met671 in the conventional $\beta$ APP mRNA, the translational outcome of the $\beta A P P$ mRNA amplification pathway would be the C99 fragment of amyloid precursor protein produced independently of $\beta A P P$ and containing $A \beta$ at its $\mathrm{N}$-terminus. Is such an outcome feasible?

Projected Pathway of Asymmetric Amplification of Human $\beta$ APP mRNA Resulting in Chimeric mRNA Encoding the C99 Fragment of Beta-Amyloid Precursor Protein
With regard to questions (1) and (2) formulated above, to determine if an mRNA species of interest can potentially be a subject of RNA-dependent mRNA amplification (provided that the cellular RdRs machinery is activated), one needs to assess whether its antisense complement contains both the TCE and the ICE and is capable of folding into a self-priming configuration. If it is, this will answer affirmatively question (1), and the position of the ICE will indicate the possible translational outcome, thus providing the answer to question (2). Such an assessment can be conducted in a model experiment where an mRNA of interest serves as a template for synthesis of cDNA, initiating at the 3'-terminal poly(A), and is subsequently removed by RNAse $\mathrm{H}$ activity present in a preparation of reverse transcriptase used. If an mRNA is fully transcribed, if complementary elements are present within the antisense strand (cDNA), if one of them is 3'-terminal, and if they are topologically compatible, i.e. mutually accessible within the folded antisense molecule, self-priming and the extension synthesis of a segment of the sense strand would occur. The junction between the antisense and sense components would define the site of self-priming and facilitate identification of the TCE and ICE. Just such an experiment was inadvertently carried out with human $\beta A P P$ mRNA [26]. The results of this experiment, misinterpreted and eventually dismissed by the authors as an artifact [27], indicated the occurrence of the topologically compatible TCE and ICE elements within the antisense strand of $\beta$ APP mRNA and defined their nucleotide sequences as well as the position of self-priming. Based on these results, the TCE/ICEguided folding and extension of the antisense strand of human $\beta$ APP mRNA [2-8] can be depicted as shown in Figure 2.

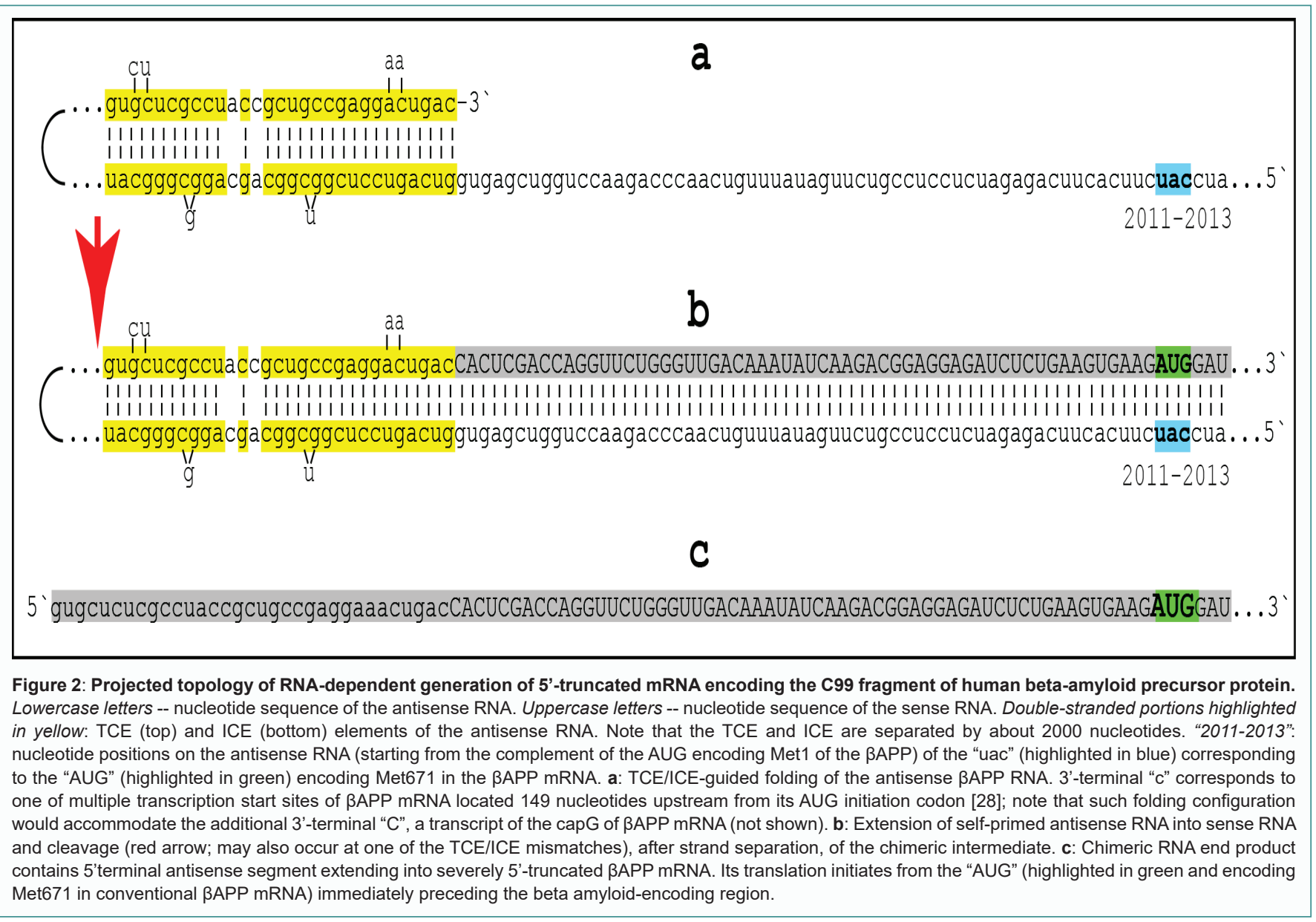


An approximately 30 nucleotide-long 3 '-terminal segment of the antisense strand of human $\beta$ APP mRNA constitutes the TCE. Its counterpart, the ICE, is separated by nearly 2000 nucleotides, yet these elements (highlighted in yellow in Figure 2) are topologically compatible and the folding of the antisense molecule results in a selfpriming configuration capable of accommodating the additional 3' $\mathrm{C}$ not encoded in the genome, a transcript of the capG of $\beta$ APP mRNA (Figure 2a). The TCE serves as a primer and is extended; thus generating the sense strand as shown in Figure $\mathbf{2} \mathbf{b}$. Strands are then separated as illustrated in Steps 5' and 6' of Figure 1, and cleavage occurs either at the mismatches within the TCE or immediately upstream as indicated by the arrow in Figure $2 \mathbf{b}$. The resulting chimeric RNA end product, shown in Figure 2 c, consists of an antisense segment (TCE or its portion) continued into a sense-orientated molecule. The translational outcome is decided by the first, 5 '-most, initiation-competent AUG codon. As shown in Figure $2 \mathbf{b}$ and $\mathbf{c}$, the first AUG codon (highlighted in green in Figure 2) is located 58 nucleotides downstream from the TCE portion of the chimeric RNA end product and it is, in fact, the AUG encoding Met671 in the intact $\beta A P P$ mRNA! Translation from this position would produce the C-terminal fragment of $\beta A P P$ containing beta-amyloid at its $\mathrm{N}$-terminus, the C99 fragment of the beta-amyloid precursor protein, in a $\beta$ APP-independent manner. The major prediction of such a mechanism is a complete inefficiency of beta-secretase inhibition in Alzheimer's disease. This prediction was, in fact, born out in several massive stage III clinical trials [5,9].

\section{In Humans, Only a Subset of $\beta$ APP mRNA Transcripts is Eligible for the RNA-Dependent Amplification Process: Transcription Start Site Usage May Contribute to Susceptibility or Resistance to AD}

The presence of a regulatory element known as a "TATA-box" is characteristic for a large class of mammalian genes. Usually, it occurs about 25 nucleotides upstream from the transcription start site (TSS) and rigidly defines its position. The mammalian $\beta$ APP gene belongs to a class of TATA-less genes that are characterized by multiple transcription start sites. There are at least five and possibly more positions where transcription of human $\beta$ APP mRNA can be initiated $[2,28]$. Of those, only one, 149 nucleotides upstream from the AUG translation initiation codon, shown in Figure 2, results in an mRNA molecule eligible for the RNA-dependent mRNA amplification process because only for this transcript would the position of the TCE on its antisense strand be strictly $3^{\prime}$-terminal $[2,28]$, and the additional 3'C, a transcript of the capG of $\beta$ APP mRNA [11-13], would be accommodated in the antisense RNA self-priming structure. Utilization of human $\beta$ APP transcription start sites can be, therefore, one of the factors that define susceptibility or resistance to Alzheimer's disease. The ability to regulate the usage of $\beta$ APP transcription start site(s), or even to shift it at will, could, therefore, open powerful therapeutic applications.

\section{Exclusivity of Alzheimer's Disease to Humans: Animal $\beta$ APP mRNA is Ineligible for Amplification}

The presence of poly(A) at the 3' terminus of an RNA makes the molecule an eligible RdRp template, but it does not necessarily make it eligible for amplification. For this, the antisense RNA strand should be capable of forming a stable self-priming structure in which its 3 ' terminus can be extended into a segment of the conventional mRNA molecule. Folding of the antisense RNA in such a self-priming configuration requires, in turn, the occurrence of two complementary and topologically compatible elements, one of which is strictly 3'-terminal. It appears that this requirement is not met in antisense RNA complements of $\beta A P P$ mRNA in non-human mammals. In animals, $\beta$ APP antisense RNA segments corresponding to the TCE and ICE elements of human $\beta A P P$ antisense RNA show little, if any, complementarity; moreover, the 3'-terminal segments of various animal antisense $\beta$ APP RNAs have no extensive complementarity with the rest of the molecule. Thus, in non-human mammals, $\beta$ APP mRNA, although a suitable RdRp template by virtue of containing 3'-terminal poly(A), is not eligible for the RNA-dependent mRNA amplification process. Therefore, if, as suggested above, the extent of increase in beta-amyloid levels sufficient to trigger a cascade of molecular events culminating in AD cannot be reached by the $\beta A P P$ proteolytic pathway alone and requires the activation of the $\beta$ APP mRNA amplification pathway, Alzheimer's disease can occur in humans but not in animals, a conclusion consistent with observations in the field.

\section{Activation of the $\beta A P P$ mRNA Amplification Pathway in Alzheimer's Disease}

Recently, it was suggested [6-8] that in Alzheimer's disease the $\beta A P P$ mRNA amplification pathway is activated by stresses associated with mitochondrial dysfunction. One type of such stresses, namely the ER stress, was previously implicated in activation of the mRNA amplification process in cells overproducing extracellular matrix proteins [13]. There is indeed a connection between mitochondrial dysfunction and ER stress [6-8]. One of the mitochondrial components, the microprotein PIGBOS, was shown to interact with the ER in mitigating the Unfolded Protein Response (UPR) [29]. It is feasible that mitochondrial dysfunction suppresses the occurrence and/or functionality of PIGBOS; this, in turn, may lead to suppression of the UPR and, consequently, to ER stress. It is therefore possible that mitochondrial dysfunction triggers the expression of inducible cofactors of the RdRp complex by initiating ER stress accompanied by activation of several transcription factors [30,31], potentially inducible components of the mRNA amplification process or the enablers of their expression. Alternatively, the expression of inducible components of the RdRp complex can be mediated by the integrated stress response apparatus $[19,20]$, which also enables the expression of some transcription factors. Importantly, mitochondrial distress was shown to be relayed to the cytosol, the locality of the ISR machinery, by an OMA1-DELE1-HRI pathway, eliciting the integrated stress response and culminating in phosphorylation of eIF2 $\alpha$, which suppresses translation of the bulk of conventionally produced mammalian mRNA species but promotes production of transcription factors such as ATF4, ATF5 and CHOP [32,33]. In this case, if, as argued above, nucleotide modifications of mRNA produced in the RNAdependent mRNA amplification pathway render it compatible with phosphorylated eIF2 $\alpha$, intracellularly retained $A \beta$ would be preferentially produced in the AD-affected neurons.

Mitochondrial dysfunction in neurons, in turn, is triggered by A $\beta$ accumulated inside the cell to sufficient levels [6-8]. Whereas the scale of intraneuronal retention of beta-amyloid postulated in these studies [6-8] is unparalleled in that, in $\mathrm{AD}$, it applies to the entire output of the predominant $\beta$ APP mRNA amplification pathway of $A \beta$ production, a notion of intracellular $A \beta$, albeit as a fraction of its secreted counterpart, is not unprecedented. There are two potential sources of intracellular $A \beta$. The more studied and understood source is cellular uptake of secreted extracellular beta-amyloid. Data obtained conclusively show that soluble $A \beta 42$ and $A \beta 40$ use endocytosis [34] to enter the cell and that $A \beta 42$ is taken up two times more efficiently 
than $A \beta 40$ [35]. Beta-sheet-rich $A \beta 42$ aggregates were observed to enter cells at low nanomolar concentrations [36]. In contrast, monomers were shown to bound to plasma membrane and to form aggregates there before cellular uptake and accumulation in endocytic vesicles [37], thus indicating that formation of $A \beta$ aggregates may be a prerequisite for cellular uptake [34,36-38]. Moreover, it was suggested that oligomer-specific $A \beta$ toxicity in cell models is mediated by its selective uptake [34]. Cellular uptake of $A \beta$ was also shown to be ApoE isoform-dependent and mediated by lipoprotein receptor LR11/SorLA [37]. ApoE4, a major genetic risk factor for AD, was shown to be much more efficient in mediating $A \beta$ uptake than ApoE3 and ApoE2 [37]. LRP, another member of the lipoprotein receptor family, binds to $A \beta$ directly or through ligands such as ApoE and undergoes endocytosis, thus facilitating cellular uptake of $A \beta$ [39]. The internalization of extracellular $A \beta$ can also be mediated by $\alpha 7$ nicotinic acetylcholine receptor [40-42], the scavenger receptor for advanced glycation, RAGE [43-45], the formyl peptide receptor-like 1, FPRL1 [46], and N-methyl-d-aspartate, NMDA, receptors [47]. $A \beta$ internalization was observed in multiple cell types, including neurons, astrocytes, glial cells and macrophages, where it appears to play different roles, and it occurs in cells of normal subjects as well as in cells of AD-affected individuals [48].

The other potential source of intracellular $A \beta$, operating only in beta-amyloid producing cells, i.e. neurons in the subject under discussion, is its retention within the cell. Whether $A \beta$ is retained intracellularly or is secreted into the extracellular pool is defined by the location at which the immediate beta-amyloid precursor, the C99 fragment, is cleaved by the gamma-secretase complex. The vast majority of $A \beta$ produced in the $\beta A P P$ proteolytic pathway is generated by cleavage at the plasma membrane and is secreted. However, cleavage can also occur in the endoplasmic reticulum (ER) [49], Golgi and trans Golgi network (TGN) [50], and at endosomal [49], lysosomal [49] and mitochondrial [51]] membranes; such cleavages generate intracellularly retained $A \beta$. It has been shown that different isoforms of intracellular $A \beta$ can be generated at different locations. For example, cleavage within the ER produces predominantly $A \beta 42$ [52-56] whereas cleavage within the TGN mostly generates $A \beta 40$ [57] Interestingly, these locations of intracellular $A \beta$ generation are limited to neurons [53]. Recent evidence suggests that subcellular localization of presenilin2 (PSEN2) directs the assembly of gamma-secretase complex to specific cellular compartments and thus contributes to the balance between intracellular accumulation and secretion of $A \beta$ $[58,59]$. Moreover, FAD-associated PSENs mutations were shown to pronouncedly augment the intracellular pool of $A \beta$ by determining localization and substrate specificity of gamma-secretase [58].

\section{Intracellular Retention of $A \beta$ Produced in the Asymmetric $\beta A P P$ mRNA Amplification Pathway Is Neuron-Specific}

Whereas only a fraction of $A \beta$ produced in the $\beta$ APP proteolytic pathway is retained intraneuronally, in the new paradigm, the entire output of the predominant $\beta A P P$ MRNA amplification pathway of betaamyloid production remains inside the $A D$-affected neurons (until they die as a result, that is). Normally, proteolytic processing of the bulk of $\beta$ APP occurs in the secretory pathway and culminates in gammasecretase cleavage within the plasma membrane and subsequent secretion of newly generated $A \beta$ into extracellular space. A nascent $\beta$ APP molecule is chaperoned into the secretory pathway through its $\mathrm{N}$-terminal signal peptide. There is, however, no N-terminal signal peptide in the translational end product of asymmetric amplification of human $\beta A P P$ mRNA. Indeed, the $\beta A P P$ mRNA amplification pathway results in the C99 fragment. C99 lacks N-terminal signal peptide and requires only gamma-secretase cleavage to produce $A \beta$. This cleavage can occur on intracellular membranes at a variety of sites, such as ER and TGN [50-55], where gamma-secretase cleavage was shown to occur only in neurons [53], and even in mitochondria where all subunits of the gamma-secretase complex were shown to be present $[57,60,61]$. If this cleavage occurs in the ER, the $A \beta 42$ isoform will be predominantly produced [53]. If both ER and TGN sites are utilized, a mixture of $A \beta 42 / A \beta 40$ will result [53]. Since the processing of the C99 fragment expressed from a construct encoding only this polypeptide, rather than $\beta$ APP, was shown to result, due to the presence of an internal transmembrane domain, in secretion of $A \beta$ in a non-neuronal cell model [21] and because cleavage on various intracellular membranes was shown to occur only in neurons, it has to be assumed that intracellular retention of $A \beta$ produced from C99-encoding $m R N A$, the end product of asymmetric $\beta A P P \quad m R N A$ amplification, is neuron-specific, a conclusion potentially instrumental, as discussed below, in construction of experimental AD models. The bottom line is that for $A \beta$ to be retained intraneuronally, its precursor, the $\mathrm{C} 99$ fragment, produced either in the proteolytic or in the ADspecific $\beta$ APP mRNA amplification pathways, has to be processed, i.e. cleaved by gamma-secretase, on an intracellular membrane. In the $\beta$ APP proteolytic pathway this occurs infrequently, whereas in the $\beta$ APP mRNA amplification pathway of $A \beta$ production, this is the only way. Moreover, there are multiple options, some generating predominantly $A \beta 42$ [52], to accomplish this. In addition, if the heavily modified mRNA end product of asymmetric human $\beta$ APP mRNA amplification, encoding the C99 fragment, is translated in a compartmentalized manner, this may also contribute to selection of gamma-secretase cleavage sites on intracellular rather than plasma membranes.

\section{Engine that Drives Alzheimer's Disease}

Intraneuronal accumulation of conventionally produced $A \beta$ to levels sufficient to trigger mitochondrial dysfunction-associated stresses and, consequently, activation of the $\beta A P P$ mRNA amplification pathway, plays the role of a starter motor in getting car engine moving in a self-sustainable manner. In the case under discussion, it activates the engine that drives AD. Indeed, stresses associated with mitochondrial distress and/or stress responses, presumably the ISR, activate the predominant, $\mathrm{AD}$-specific, pathway of $\mathrm{A} \beta$ generation, the $\beta A P P$ mRNA amplification process [6-8]. The entire output of this pathway is retained intraneuronally. Drastically increased levels of intracellular $\mathrm{A} \beta$ promote, in turn, further mitochondrial dysfunction and associated stresses/stress responses and, consequently, the $\beta$ APP mRNA amplification process, thus generating self-perpetuating betaamyloid overproduction/mitochondrial dysfunction mutual feedback cycles. These relationships are diagrammatically summarized in Figure 3. It depicts the mutual feedback cycles as a two-stroke engine, the engine that drives beta-amyloid overproduction and, consequently, Alzheimer's disease; ultimately, it triggers neuronal death. To develop sporadic $\mathrm{AD}$, it takes, apparently, a lifetime of intraneuronal accumulation of conventionally produced $A \beta$ to critical levels sufficient to trigger mitochondrial dysfunction and associated stresses/ stress responses, combined with the susceptibility to the disease (e.g. appropriate TSS usage permitting operation of the $\beta$ APP mRNA amplification pathway). In familial $\mathrm{AD}$, because of abnormal $\beta \mathrm{APP}$ proteolytic processing, critical intraneuronal levels of conventionally produced $\mathrm{A} \beta$, or of its more "toxic" isoforms with increased capacity to trigger mitochondrial dysfunction and/or to aggregate, are reached sooner and the disease occurs earlier in life. 


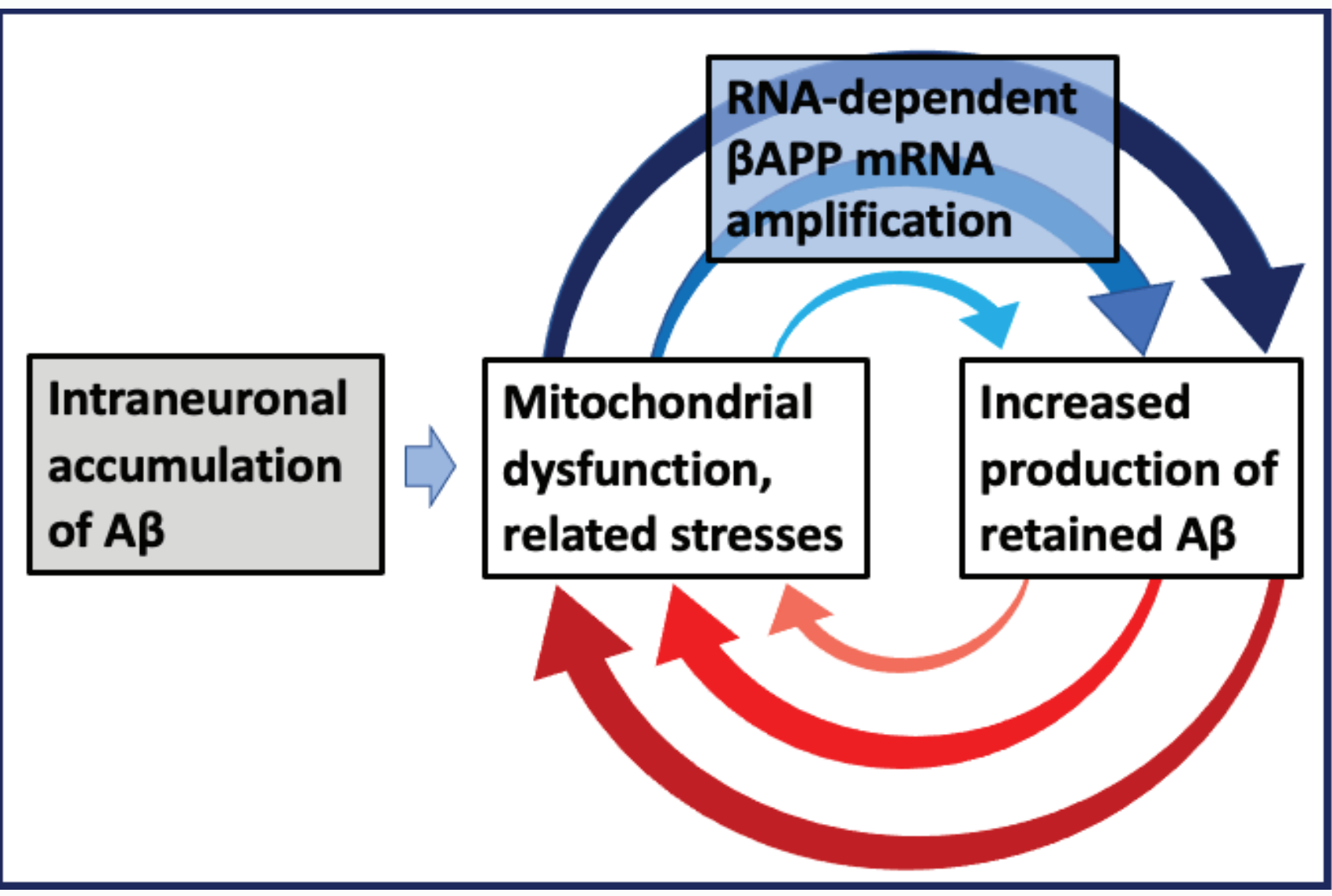

Figure 3: The engine that drives AD: Self-propagating mutual feedback cycles of mitochondrial dysfunction-mediated overproduction of $A \beta$ and vice versa in Alzheimer's disease. Highlighted in grey: Intraneuronal accumulation of $A \beta$ via both cellular uptake of secreted peptide and retention of a fraction of beta-amyloid produced in the $\beta$ APP proteolytic pathway; Highlighted in blue: Asymmetric RNA-dependent $\beta A P P$ mRNA amplification, a molecular basis of A $\beta$ overproduction in Alzheimer's disease. Horizontal arrow: Intraneuronal accumulation of $A \beta$ to critical levels acts as a starter motor that initiates the self-sustainable engine that drives AD. Arched arrows: Self-propagating mutual feedback cycles; Red arches: Intraneuronal A $\beta$-mediated induction of mitochondrial dysfunction and related stresses; Blue arches: Mitochondrial dysfunction/related stresses-mediated RNA-dependent amplification of $\beta A P P$ mRNA resulting in overproduction of intraneuronally retained $A \beta$.

Alzheimer's Disorder is a Fast Disease Preventable by Therapeutic Intervention Initiated Even Late in Life, and Treatable and Reversible at the Early Symptomatic Stages

The new paradigm posits that Alzheimer's disorder is a "fast" disease. This is in sharp contrast to a view, prevailing until now, that $\mathrm{AD}$ is a quintessential "slow" disease that develops throughout the life as one prolonged process. Dynamics of the disease in the old paradigm is presented in panels $\mathbf{A}$ and $\mathbf{B}$ of Figure 4. It is single-phased and can be divided into asymptomatic (red lines) and symptomatic (red blocks) portions. $A \beta$, it is assumed, is overproduced (and secreted) solely in the $\beta$ APP proteolytic/secretory pathway. As its extracellular levels increase, it triggers neurodegeneration (red lines) starting early in life. Damages accumulate and manifest symptomatically (red blocks) late in life in sporadic cases (panel A). In familial AD cases, where mutations in the $\beta$ APP gene or in presenilins increase production of either common $A \beta$ isoform or of its more toxic isoforms, neurodegeneration reaches critical threshold sooner and $\mathrm{AD}$ symptoms occur earlier in life, mostly in the late 40s and 50s (panel B). The disease is considered untreatable in the symptomatic phase and there are currently no preventive $\mathrm{AD}$ therapies, but if they were available, according to this viewpoint, it would be largely futile to intervene late in life in case of potential SAD or at mid-age in cases of FAD because, although $\mathrm{AD}$ symptoms have not yet manifested, the irreversible damage has already occurred during the preceding decades. In this paradigm, to be effective, preventive therapeutic intervention should be initiated early and continued for life.

Dynamics of the disease in the new paradigm, illustrated in panels C (SAD) and D (FAD), is radically different [6-8]. This dynamic is biphasic. In the first phase, only the $\beta$ APP proteolytic pathway of $A \beta$ production is in operation. This phase is a slow process of intraneuronal beta-amyloid accumulation. It occurs via cellular uptake of secreted $A \beta$ and retention of a fraction of $\beta$ APP-derived $A \beta$. These processes are common to Homo sapiens, including healthy humans, and to non-human mammals, and result neither in significant damage (black lines), nor in any manifestation of the disease; there is, in fact, no disease. The second phase occurs exclusively in humans and commences with the $\mathrm{A} \beta$-induced mitochondrial dysfunction which, in turn, mediates the activation of the $\beta A P P$ mRNA amplification pathway shortly before symptomatic onset of the disease. In this phase, the rate of production and the extent of intraneuronal accumulation of retained $A \beta$ sharply accelerate, causing, in conjunction with mitochondrial dysfunction, after a lag period when amplified C99encoding RNA accrues and intraneuronal $A \beta$ further accumulates (black lines), significant neurodegeneration (red lines) and triggering $\mathrm{AD}$ symptoms (red blocks); this phase is fast. In this paradigm, a preventive therapy for $A D$, an $A D$ "statin", would be effective when initiated at any time prior to commencement of the second phase 


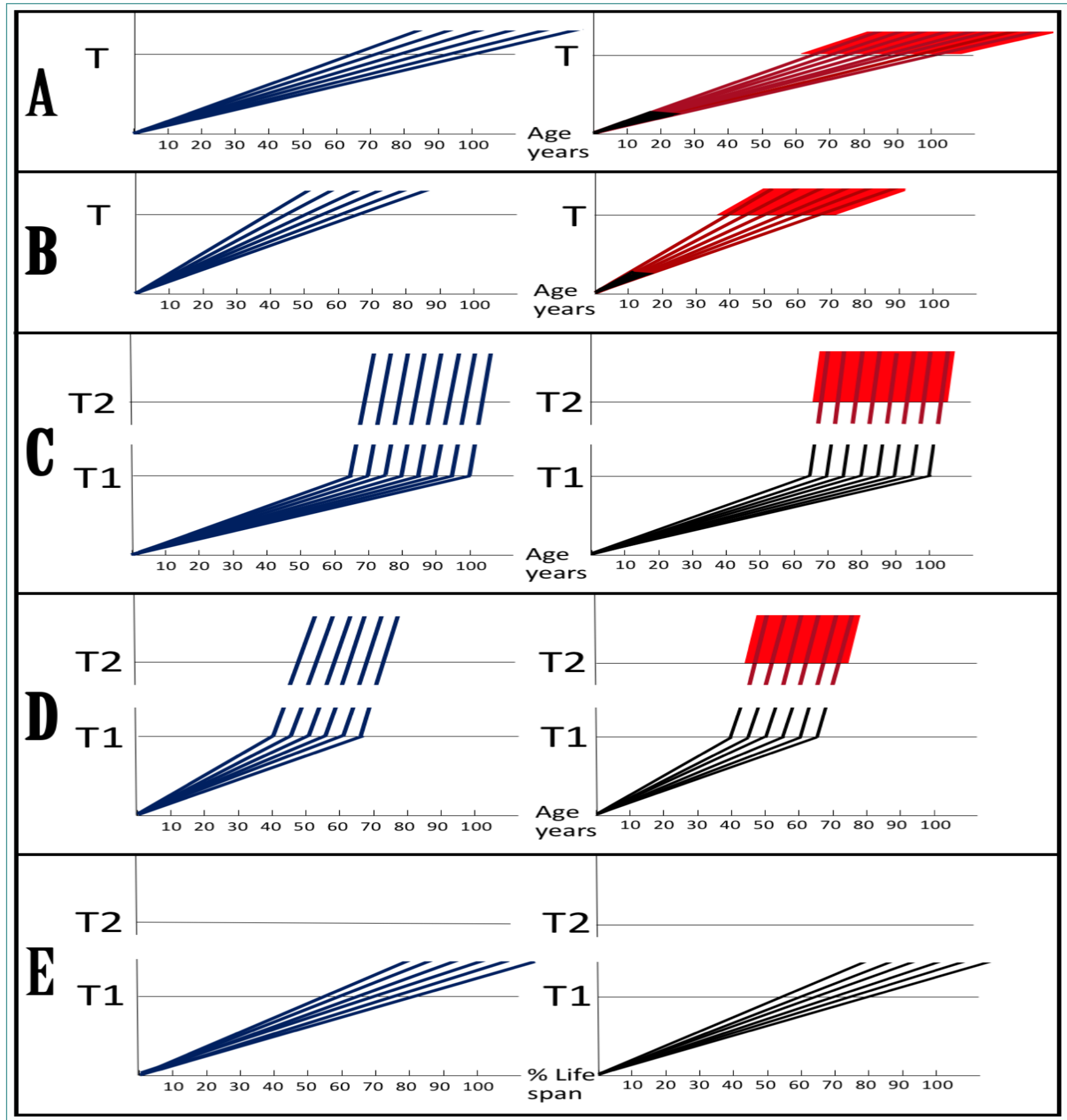

Figure 4: Dynamics of Alzheimer's disease in two paradigms. Left panels: Dynamics of A $\beta$ production; Right panels: Dynamics of neurodegeneration. Blue lines: Levels of beta-amyloid; Red lines: Extent of neurodegeneration; Black lines: Indicator lines, no significant neurodegeneration; Red blocks: Symptomatic manifestation of $A D$. T: Threshold of symptomatic manifestation of $A D$ (reflects levels of extracellular $A \beta$ and consequent extent of neurodegeneration); $T 1$ : Threshold of activation of RNA-dependent $\beta A P P$ mRNA amplification (reflects both intraneuronal $A \beta$ levels and the consequent extent of $A \beta-$ coupled mitochondrial dysfunction); Numerous genetic factors such as the occurrence of various alleles of ApoE gene, as well as certain epigenetic factors, influence the age when thresholds $\mathrm{T}$ and $\mathrm{T} 1$ are reached, hence, the fanning lines.T2: Threshold of symptomatic occurrence of $A D$ (reflects levels of intraneuronal $A B$,degree of mitochondrial dysfunction and consequent extent of neurodegeneration). Panels $\mathbf{A}, \mathbf{B}$ : View of the dynamics of $A D$ in the old paradigm (A: Dynamics of SAD; $\mathbf{B}$ : Dynamics of FAD). Levels of extracellular $A B$ increase, neurodegeneration starts early and accumulates throughout the life. When threshold $T$ is reached, $A D$ symptoms manifest. Panels $\boldsymbol{C}, \boldsymbol{D}$ : The outlook on the dynamics of $A D$ in the new paradigm (C: Dynamics of SAD; D: Dynamics of FAD). Levels of intraneuronal A $\beta$ increase, the extent of mitochondrial dysfunction and related stresses reach threshold T1 and RNA-dependent $\beta A P P$ mRNA amplification is activated. There is no significant neurodegeneration until after a lag period (when amplified RNA encoding the C99 fragment of $\beta A P P$ accrues and intraneuronal A $\beta$ further accumulates) following the crossing of T1 threshold and activation of BAPP mRNA amplification; when the extent of neurodegeneration reaches threshold T2, AD symptoms manifest. Panel E: Dynamics of A $\beta$ production and neurodegeneration in non-human mammals: T1 threshold is crossed but $\beta A P P P$ mRNA is not amplified because it is not eligible for RNA-dependent amplification process. There is no significant neurodegeneration; T2 threshold is not reached, no AD symptoms manifest, no disease occurs. Note: Scenario depicted in panel(E) would occur in humans not susceptible to Alzheimer's disease due to variations in $\beta A P P$ TSSs utilization or for other reasons, or when the $\beta A P P$ mRNA amplification pathway of A $\beta$ production is effectively interfered with by therapeutic intervention. 
(panel E). Moreover, there are good reasons to believe that with a drug blocking the $\beta A P P$-independent $A \beta$ production pathway, asymmetric $\beta$ APP mRNA amplification, or effectively interfering with $\mathrm{A} \beta$ production, it would be possible not only to preempt the disease but also to stop and reverse it even when early AD symptoms have already manifested. This assumption is based on observations in studies where $\mathrm{AD}$ symptoms, as well as neurodegeneration, were reversed by suppressing, via BACE inhibition, overproduction of beta-amyloid in mouse $\mathrm{AD}$ models generating $\mathrm{A} \beta$ solely in the $\beta \mathrm{APP}$ proteolytic pathway $[62,63]$.

\section{Therapeutic Approaches for Alzheimer's Disease: Targeting the Engine}

Within the framework of the new paradigm of $\mathrm{AD}$, several potential therapeutic approaches, targeting either the immediate driver of the diesease, $A \beta$ production in the $\beta$ APP mRNA amplification pathway, or the second component of the "engine", mitochondrial dysfunction plus associated cellular stresses and/or stress responses, that activate it, can be considered. One approach is to suppress the enzymatic machinery of RNA-dependent mRNA amplification. Another is to interfere with amplification at the level of $\beta A P P$ mRNA or its antisense RNA counterpart. The third approach is to block the activation of the $\beta A P P$ mRNA amplification pathway by mitigating mitochondrial dysfunction and/or related stresses. The fourth approach is to interfere with the processing of the $\mathrm{C} 99$ fragment produced in both, the $\beta \mathrm{APP}$ proteolytic pathway, and the $\beta$ APP mRNA amplification pathway. The first approach, suppression of RdRs enzymatic components, however tempting, is not feasible. This is because systemically targeting any enzymatic component of mammalian RNA-dependent mRNA amplification would interfere with normal physiological functions of this process [11-13] and would have deleterious consequences.

The second approach, manipulation of $\beta \mathrm{APP}$ mRNA or of its antisense complement, is very promising. One way to implement it is to shift the TSS of BAPP mRNA to such a position as to make the resulting RNA molecules ineligible for the amplification process either by eliminating its 5 '-terminal segment corresponding to the TCE or making it non-terminal. Another way is to bind a small molecule to the amplified $\beta A P P$ mRNA, possibly targeting, in a sequence-specific manner or via specific delivery to neurons, its modified nucleotides, in such a way as to interfere with its function as a template in antisense RNA synthesis and/or in translation. Antisense $\beta$ APP RNA can also be specifically targeted, with the aim to disrupt its folding into the selfpriming configuration. This approach would suppress amplification and/or function of $\beta$ APP mRNA but would not affect RNA-dependent mRNA amplification machinery in general; it remains to be developed.

The third approach, suppressing activation of the $\beta$ APP mRNA amplification pathway, is, apparently, feasible. $\beta A P P$ mRNA amplification-enabling potential denotes intraneuronal $A \beta$-initiated mitochondrial dysfunction/associated cellular stresses as a possible therapeutic target. This potential is, plausibly, neuron-specific. This is, at least in part, because retention of $\beta A P P$-derived $A \beta$, instrumental in the initial activation of mitochondrial dysfunction, occurs in brain solely in neurons, the only beta-amyloid producing cells, and because, in $\mathrm{AD}$, the "engine" sustaining mitochondrial dysfunction operates also only in neurons. Moreover, if the aim is to pursue therapeutically the $\beta A P P$ mRNA amplification process as the probable molecular basis of $A \beta$ overproduction in $A D$, mitochondrial dysfunction and cellular stress/stress response components, ostensive triggers of this process, are the targets of choice. Indeed, whereas systemic targeting of the enzymatic components of RdRs would have deleterious consequences, successfully targeting mitochondrial dysfunction would potentially result in two beneficial outcomes; namely, switching off the detrimental $\beta$ APP mRNA amplification process in neurons and repairing metabolic defects. In this respect, a recent study with C. elegans [64] is very encouraging. In this study, beta-amyloid-induced mitochondrial dysfunction was modeled and achieved by expressing human A $\beta$ specifically in neurons (GRU102). Importantly, treatment with an anti-diabetes drug, metformin, reversed $A \beta$-induced metabolic defects, reduced protein aggregation and normalized lifespan of GRU102; thus establishing metabolic mitochondrial dysfunction as a promising and feasible therapeutic intervention target in AD. Targeting components of the integrated stress response or of mitochondrial dysfunction-associated stresses might also have beneficial outcomes in AD.

Whereas neither targeting extracellular beta-amyloid by immunotherapy or otherwise nor BACE inhibition can affect in any way $A \beta$ production in the $\beta$ APP mRNA amplification pathway and its resulting intraneuronal accumulation, inhibition of gammasecretase cleavage should be highly effective in suppressing betaamyloid generation in both, the $\beta$ APP proteolytic and the $\beta A P P$ mRNA amplification, pathways by blocking processing (gamma-site cleavage) of the C99 fragment. However, as was shown in several human clinical trials, gamma-secretase, a key component of the Notch signaling pathway, cannot be inhibited systemically without deleterious consequences. On the other hand, the fourth approach, utilization of known or novel modulators of gamma-secretase-mediated C99 cleavage, might, possibly, elicit therapeutic benefits in AD by shifting the isoform pattern of resulting intraneuronal $A \beta$ toward less harmful species with reduced capacity to trigger mitochondrial dysfunction. If effective, this type of $\mathrm{AD}$ therapy can be used preventively and may prove efficient as a treatment at the early symptomatic stages of the disease. The efficacy of such modulators in $\mathrm{AD}$, however, remains to be established.

\section{Experimental AD Models for the New Paradigm}

Experimental AD models for the new paradigm are very different from the existing ones. In light of the above considerations, the current $A D$ models are inadequate because they are lacking the $\beta A P P$ mRNA amplification pathway of beta-amyloid production, the major driver of the disease. The reason for this is not the lack of enzymatic apparatus but ineligibility of both endogenous and transgenic $\beta$ APP mRNAs for the RNA-dependent amplification process [6-8] as well as the lack of means for accelerated activation of the enzymatic machinery of the mRNA amplification pathway. Therefore, one objective in designing a new generation of $\mathrm{AD}$ models is to express human A $\beta$-encoding $m R N A$ eligible for the amplification process according to the criteria formulated above. Another objective is to produce beta-amyloid in such a manner that the entire output is retained intraneuronally. This is to maximally accelerate induction of mitochondrial dysfunction and related stresses and/or the ISR, and consequent activation of the BAPP mRNA amplification pathway of beta-amyloid production. Accordingly, the expressed mRNA should encode $A \beta 42$ or another preferred isoform of beta-amyloid, or the C99 fragment of $\beta$ APP containing, if needed, desired mutations; for example those increasing production of $\mathrm{A} \beta 42$ (this may not be needed if, as was argued above, neuronal processing of $\mathrm{C} 99$ derived independently of $\beta$ APP results preferentially in $A \beta 42$ ). Provided the intracellular retention of $A \beta$, resulting from processing of the $\mathrm{C} 99$ fragment produced in the RNAdependent amplification pathway independently of $\beta \mathrm{APP}$, is not 


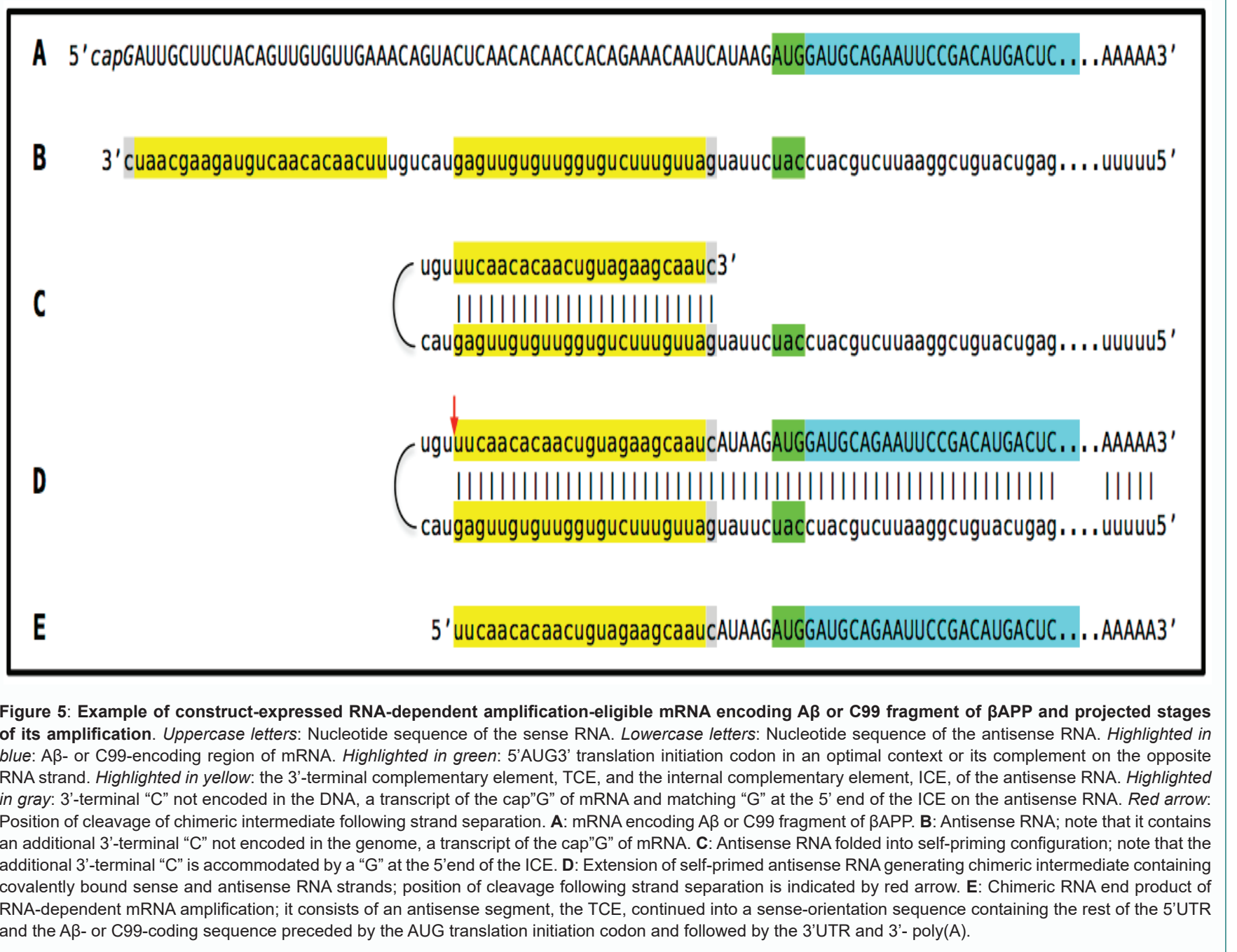

restricted to potentially compartmentalized translation product of modified RNA end product of amplification and is neuron-specific, $a$ construct expressing the $C 99$ fragment of $\beta A P P$, rather than $A \beta$, should be used in $A D$ models. This is because in such models, intracellularly retained $A \beta$, more deleterious to cells than secreted extracellular betaamyloid, would be produced only in neurons.

A generic example of a construct-expressed mRNA, encoding human $A \beta$ or, preferably, as argued above, $C 99$ and eligible for the RNA-dependent mRNA amplification process, is presented in Figure 5. This mRNA transcript contains coding information (Figure $5 \mathbf{A}$, highlighted in blue) either for the C99 fragment of human $\beta$ APP or for a defined isoform of human $A \beta$, preceded immediately and in-frame by the AUG codon (Figure $5 \mathbf{A}$, highlighted in green) in an optimal translation initiation context. Its 5 ' untranslated region, UTR, is designed in such a way that the corresponding segment of the antisense RNA contains two complementary elements, the 3'-terminal TCE and the internal ICE (Figure 5B, highlighted in yellow). Accordingly, upon its formation, the antisense RNA folds into a self-priming configuration (Figure 5C). Importantly, the sequence of the ICE is such that it can accommodate the additional 3'-terminal "C", not encoded in the DNA but a transcript of the capG of mRNA (both 3'-terminal "C" and matching "G" at the 5' end of the ICE are highlighted in gray). Such a self-priming structure is then 3 '-extended (Figure 5D) generating a sense-strand nucleotide sequence which terminates with 3' poly(A). This is followed by strand separation (not shown) and cleavage at the 5 ' end of the TCE (Figure 5D, red arrow). The resulting functional chimeric RNA end product of amplification (Figure 5E) consists, in its 5' portion, of an antisense segment, the TCE, continued into a sense-orientation sequence containing the rest of the 5'UTR and the A $\beta$ - or C99-coding sequence, preceded by the AUG translation initiation codon and followed by the 3'UTR and poly(A), and can be translated into $\mathrm{A} \beta$ or $\mathrm{C} 99$ polypeptides.

Some interesting outcomes can be attained by designing human $\mathrm{A} \beta$-encoding constructs in such a way that beta-amyloid is produced only in the RNA-dependent amplification pathway. An example of RNA transcribed from such a construct is presented in Figure 6. In it, the AUG codon, immediately preceding the human A $\beta$ - or C99coding segment (line $\mathbf{A}$, highlighted in blue) in the endogenous human $\beta A P P$ gene, is deleted and replaced with the "ACA" (its antisense counterpart, 3'ugu5', being able to accommodate 5'aug3' in the folded self-primed antisense RNA, as shown in line $\mathbf{C}$ ). There is also no functional translation initiation codon in-frame with and upstream from the $A \beta$-coding segment. RNA transcribed from such a construct contains $\mathrm{A} \beta$ - or $\mathrm{C} 99$-coding information but it cannot be translated into a polypeptide; such RNA transcript is translationally "silent" (line A). The construct is designed so that this AUG-deficient, A $\beta$ - or C99-encoding RNA is, nevertheless, eligible for the RNAdependent amplification process. Its antisense RNA transcript 


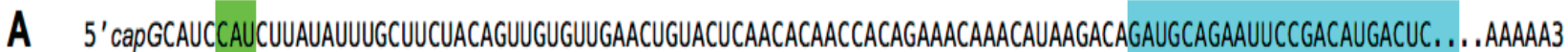

Figure 6: Example of "silent" RNA transcript from a construct producing A $\beta$ or C99 fragment solely in the RNA-dependent amplification pathway; projected amplification stages resulting in functional chimeric mRNA encoding A $\beta$ or C99. Uppercase letters: Nucleotide sequence of the sense RNA. Lowercase letters: Nucleotide sequence of the antisense RNA. Highlighted in blue: Aß- or C99-encoding region of mRNA. Highlighted in green: 5'AUG3' in an optimal translation initiation context or its complement on the opposite RNA strand. Highlighted in yellow: 3'-terminal complementary element, TCE, and internal complementary element, ICE, of the antisense RNA. Highlighted in gray: 3'-terminal "C" not encoded in the DNA, a transcript of the cap"G" of mRNA and matching "G" at the 5' end of the ICE on the antisense RNA. Red arrow: Position of cleavage of chimeric intermediate following strand separation. A-Translationally silent RNA encoding A $\beta$ or $C 99$ fragment of $\beta A P P$; note that the "AUG" immediately preceding A $\beta$-coding in endogenous mRNA has been removed and replaced by the "ACA", and there is no functional translation initiation codon in-frame with and upstream from A 3 -coding segment. B - Antisense RNA; note that it contains 5'aug3' followed in the 3' direction by sequence encoding N-terminus of A 3 at its 3'end. C - Antisense RNA folded into self-priming configuration; note that 5'aug3' is accommodated by the 3'ugu5', a complement of the "ACA" in the sense strand. D - Extension of self-primed antisense RNA generating chimeric intermediate containing covalently bound sense and antisense RNA strands; position of cleavage following strand separation is indicated by red arrow. E - Chimeric RNA end product of RNA-dependent mRNA amplification; note that it consists of the antisense portion encoding the "aug" translation initiation codon in optimal translation initiation context and the N-terminus of resulting polypeptide (A or C99), whereas the sense RNA portion encodes the rest of a polypeptide and contains 3 'UTR and 3'-terminal poly(A).

contains the 3'-terminal TCE and the internal ICE sequences (highlighted in yellow). Moreover, the TCE contains a 5'aug3' codon (highlighted in green and shown in 3' to 5' orientation) in an optimal translation initiation context (line B). Following folding into a selfpriming configuration (line $\mathbf{C}$ ), the structure is extended, strands are separated as described above and cleaved (red arrow) at the 5 ' end of the TCE (line $\mathbf{D}$ ). In the chimeric RNA end product of amplification (line E), the TCE-derived "aug" codon, highlighted in green, ends up immediately and contiguously upstream from the A $\beta$ - or C99-coding sequence (highlighted in blue) and can initiate its translation. It should be noted that in this design, the antisense strand contains not only the 5'AUG3' codon but also a segment of $A \beta$-coding sequence at its 3' terminus. This is to ascertain that the additional 3'-terminal "C" of the antisense RNA, not encoded in the DNA but a transcript of the 5'cap " $G$ " of the sense-strand RNA [10-12], can be accommodated in the folded self-priming configuration (both 3'-terminal " $\mathrm{C}$ " of the antisense RNA and matching "G" at the 5' end of the ICE are highlighted in gray). Consequently, in the chimeric RNA end product (line $\mathbf{E}$ ), the antisense portion encodes the "aug" translation initiation codon and the $\mathrm{N}$-terminus of the resulting polypeptide (A $\beta$ or $\mathrm{C} 99$ ), whereas the sense RNA portion encodes the rest of a polypeptide and contains 3'UTR and 3'poly(A). With this construct, beta-amyloid will be produced, directly or through the processing of the C99 fragment, only if and when the RNA-dependent mRNA amplification process is activated.
The obtainability of such construct opens the possibility for generation of a mouse $\mathrm{AD}$ model resulting from breeding of two mouse lines, one containing an $A \beta$-encoding construct that can produce beta-amyloid only in the RNA-dependent mRNA amplification pathway, and another, conventionally expressing, from a standard construct, intraneuronally retained $A \beta$. Only progeny expressing $A \beta$ both from a construct described above, in the RNAdependent $\beta$ APP mRNA amplification pathway, and conventionally from a standard construct, with the former activated by the latter, is expected to develop symptomatic $\mathrm{AD}$, with parental lines constituting the controls. Alternatively, both types of constructs can be inserted simultaneously in the same animal. Considerations of preferential expression of the C99 fragment rather than $A \beta$, discussed above, apply also to these AD models.

\section{Universal Reporter for the RNA-Dependent mRNA Amplification Pathway}

The concept of a construct expressing beta-amyloid only in the mRNA amplification pathway can be generalized by substituting the $\mathrm{A} \beta$-encoding portion of the construct with that encoding a tag peptide or even a fluorescent polypeptide. Such a construct would constitute a universal reporter of activity of the RNA-dependent $m R N A$ amplification process in any system. A generic example of such a reporter constructexpressed mRNA, encoding a tag peptide that is produced only in the 


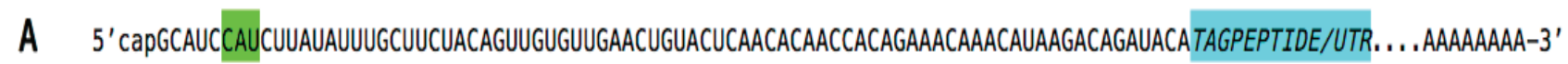

B $\quad 3^{\prime}$ 'cguagguagaauauaaacgaagaugucaacacaacuugacaugaguuguguuggugucuuuguuuguauucugucuaugutagpeptide/utr.... uuuuuuuu-5'

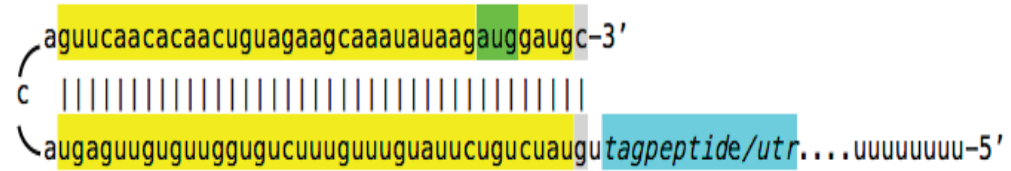

D

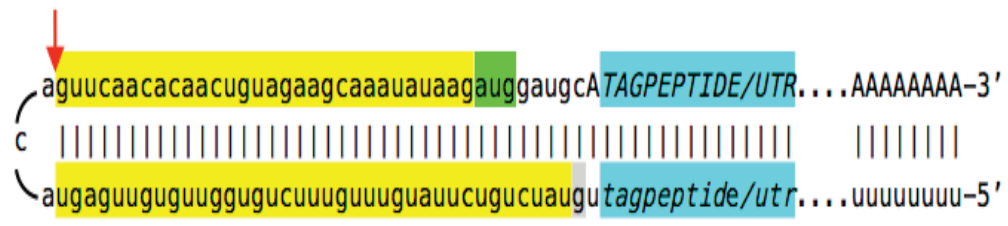

Figure 7: Example of "silent" RNA transcript from a reporter construct producing tag peptide only when the RNA-dependent mRNA amplification pathway is operational; projected stages leading to generation of functional chimeric mRNA encoding tag peptide. Uppercase letters: Nucleotide sequence of the sense RNA. Lowercase letters: Nucleotide sequence of the antisense RNA. TAGPEPTIDE/UTR, highlighted in blue, denotes nucleotide sequence encoding a tag peptide followed by 3'UTR and lacking the AUG translation initiation codon; tagpeptide/utr, highlighted in blue, denotes antisense complement of the TAGPEPTIDE/UTR nucleotide sequence. Highlighted in green: 5'AUG3' in an optimal translation initiation context or its complement on the opposite RNA strand. Highlighted in yellow: the 3'-terminal complementary element, TCE, and internal complementary element, ICE, of the antisense RNA. Highlighted in gray: 3'-terminal "C" not encoded in the DNA, a transcript of the cap"G" of mRNA and matching "G" at the 5' end of the ICE on the antisense RNA. Red arrow: Position of cleavage of chimeric intermediate following strand separation. A - Translationally silent RNA transcript encoding tag peptide and lacking the AUG translation initiation codon; there is also no functional translation initiation codon upstream from and in-frame with the tag peptide-encoding segment. B - Antisense RNA; note that it contains 5'aug3' in the optimal translation initiation context. C - Antisense RNA folded into self-priming configuration; note that 5'aug3' is accommodated by the 3'ugu5', a complement of the "ACA" in the sense strand. D - Extension of self-primed antisense RNA generating chimeric intermediate containing covalently bound sense and antisense RNA strands; position of cleavage following strand separation is indicated by red arrow. E - Chimeric mRNA end product of RNAdependent mRNA amplification; note that the "aug" translation initiation codon in optimal translation initiation context provided by the antisense portion is in-frame with the tag peptide-encoding nucleotide sequence in the sense portion of the chimeric RNA end product.

RNA-dependent amplification pathway is shown in Figure 7. In it, TAGPEPTIDE/UTR highlighted in blue denotes nucleotide sequence encoding a tag peptide followed by 3'UTR and lacking the AUG translation initiation codon. There is also no functional translation initiation codon upstream from and in-frame with the tag peptideencoding segment; no polypeptide can be translated from this RNA, it is translationally "silent" (line A). In the corresponding antisense RNA (line B), tagpeptide/utr, highlighted in blue, denotes antisense complement of the TAGPEPTIDE/UTR nucleotide sequence; it also contains the 3 '-terminal TCE and the internal ICE sequences (both highlighted in yellow). Moreover, the TCE contains a 5'aug3' codon (highlighted in green and shown in $3^{\prime}$ to $5^{\prime}$ orientation) in an optimal translation initiation context. Following the antisense RNA folding into a self-priming configuration (line $\mathbf{C}$ ), the structure is extended, strands are separated and the cleavage occurs (red arrow) at the 5' end of the TCE (line D). In the chimeric RNA end product of amplification (line E), the TCE-derived "aug" codon, highlighted in green, is upstream from and in-frame with the tag peptide-coding sequence and can initiate its translation. The design of the construct ascertains that the additional 3'-terminal " $\mathrm{C}$ " of the antisense RNA, not encoded in the DNA but a transcript of the 5'cap " $G$ " of the sense-strand RNA [11-13], can be accommodated in the folded selfpriming configuration (both 3 '-terminal " $\mathrm{C}$ " of the antisense RNA and matching "G" at the 5' end of the ICE are highlighted in gray).
With this construct, tag peptide is produced only if and when the RNAdependent mRNA amplification pathway is operational; its occurrence reports the activity of the pathway.

\section{Reporter-Based Optimal Experimental AD Models}

The feasibility of a reporter for the RNA-dependent mRNA amplification pathway suggests a blueprint for the construction of, arguably, an optimal cell-based model of AD. In this design, human iPSCs are stably transfected with a DNA construct of a type depicted in Figure 7. It encodes a tag peptide that can be produced only if construct-transcribed RNA is amplified in the RNA-dependent mRNA amplification pathway and the AUG codon, capable of initiating the tag peptide translation, is contributed to the chimeric RNA end product of amplification by its antisense component. The occurrence of the tag peptide, therefore, reports the activity of the RNA-dependent mRNA amplification pathway. The resulting modified iPSCs are differentiated into neurons and the neuronal cultures are transiently transfected by amplification-ineligible RNA encoding human $\mathrm{A} \beta 42$ or $\mathrm{C} 99$ (transiently transfected DNA requires cell division for its expression). If necessary, cells may be transfected multiple times at few day intervals; A $\beta 42$ may also be delivered into the neurons by other feasible means. Intracellular accumulation of $A \beta 42$, translated (or C99-derived) from transiently transfected RNA or delivered by other means, to levels sufficient to trigger mitochondrial 
dysfunction and associated stresses and/or stress responses is expected to lead to the activation of the RNA-dependent mRNA amplification pathway which, in turn, would result in production of the tag peptide, a reporter for this pathway. Such a human cell-based system, enabling a simple and efficient assay, would be instrumental both in studying $A \beta$-mediated activation of the mRNA amplification pathway and in testing potential drugs inhibiting the initiation of this process. Moreover, the eligibility of human $\beta$ APP mRNA for asymmetric amplification (subject to the TSS usage as stipulated above and verifiable by the occurrence of rigorously predictable and uniquely specific antisense/ sense RNA junction sequences [11-13]) would result in generation of endogenous C99 produced via this process independently of $\beta$ APP and processed, in a neuron-specific manner, into intracellularly retained beta-amyloid enriched in $\mathrm{A} \beta 42$ isoform. This, in turn, would, in conjunction with mitochondrial dysfunction and without additional transient transfections or other $A \beta$ deliveries, sustain the activity of the $\mathrm{AD}$-driving engine, i.e. beta-amyloid overproduction/ mitochondrial dysfunction mutual feedback cycles, further propagate the production of endogenous $A \beta$ via $\beta A P P$ mRNA amplification pathway, and potentially lead to manifestation of AD features, such as neurofibrillary tangles [65], that can be evaluated in assessing the efficiency of candidate drugs. The employment of iPSCs derived from Alzheimer's patients would strongly increase the probability that the TSS(-149) is utilized and, therefore, that endogenous $\beta$ APP mRNA is amplification-eligible. In this system, production of $A \beta$ in the RNAdependent mRNA amplification pathway would be insensitive to BACE inhibitors but suppressed by gamma-secretase inhibitors and affected, in its isoform pattern, by gamma-secretase modulators.

In an unlikely case that in the above iPSCs-originated models, the TSS utilization pattern is such that it makes endogenous $\beta$ APP mRNA ineligible for the RNA-dependent amplification process, an alternative design variant can be employed. In it, human iPSCs are stably transfected with a DNA construct shown in Figure 6 and encoding human beta-amyloid or C99 fragment of $\beta$ APP with A $\beta$ segment tagged by one of the FAD-associated mutations. This is to distinguish, upon modified iPSCs' differentiation into neurons and activation of the RNA-dependent mRNA amplification pathway by A $\beta$-encoding RNA transfections, the resulting construct-derived beta-amyloid from that produced endogenously and from $\mathrm{A} \beta$ molecules translated from transiently transfected RNA. In this variant, construct-derived, mutation-tagged $A \beta$ itself serves as a reporter of the activity of the RNAdependent mRNA amplification pathway, in addition to its functional role. Once activated, expression from the construct would result in intraneuronally retained beta-amyloid that will sustain the activity of self-perpetuating $A \beta$ overproduction/mitochondrial dysfunction mutual feedback cycles and trigger manifestation of the $\mathrm{AD}$ features even in the absence of endogenous $\beta$ APP mRNA amplification.

Whereas all animal $A D$ models are intrinsically compromised to a certain degree because they express, non-physiologically, beta-amyloid in multiple cell types and at various developmental stages, human iPSCs-originated, cell-based experimental models described above are, arguably, the optimal ones because, unlike other experimental systems, they allow differentiation of stem cells into neurons, unimpeded and unaffected by non-physiological production of beta-amyloid or any other protein in undifferentiated precursor cells.

\section{Conclusion}

In summary, the paradigm described above introduces a new theory of Alzheimer's disease. It explains highly discordant results obtained in animal studies versus outcomes of human clinical trials, defines distinct therapeutic targets, provides blueprints for a new generation of conceptually novel AD models and specifies design of a universal reporter for the RNA-dependent mRNA amplification pathway. Most importantly, after years of despair, it offers detailed guidance and tangible hope for prevention of the disease and its effective treatment at the early symptomatic stages.

\section{Authors Contributions}

V.V. conceived and developed underlying concepts, designed animal and cell-based models of Alzheimer's disease as well as a reporter for the RNA-dependent mRNA amplification pathway described in the present study, and wrote the manuscript. S.R. contributed to development of the concepts and to design of $\mathrm{AD}$ models. B.O. participated in discussions unrelated to the design of a reporter construct and $\mathrm{AD}$ models.

\section{Funding \\ NIH R21 GM056179; NIH RO1 AR036819. \\ Conflicts of Interest}

Authors declare no conflict of interest.

\section{References}

1. Conan-Doyle A. The sign of the four. Lippincott's Monthly Magazine. 1890.

2. Volloch V. A mechanism for B-amyloid overproduction in Alzheimer's disease: Precursor-independent generation of $B$-amyloid via antisense RNA-primed mRNA synthesis. FEBS Lett. 1996;390:124-8.

3. Volloch V. Mechanism for ß-amyloid overproduction in sporadic Alzheimer's Disease: Possible antisense RNA-mediated generation of a 5'-truncated BAPP mRNA encoding $12 \mathrm{kDa} C$-terminal fragment of $\mathrm{BAPP}$, the immediate precursor of $\mathrm{A}$. In: Molecular Mechanisms of Dementia. 1997, W. Wasco and R. Tanzi, Eds.

4. Volloch V. Possible mechanism for resistance to Alzheimer's disease (AD) in mice suggests new approach to generate a mouse model for sporadic $\mathrm{AD}$ and may explain familial resistance to AD in man. Exp Neurobiol. 1997;144:214-8.

5. Volloch V, Rits S. Results of beta-secretase-inhibitor clinical trials support amyloid precursor protein-independent generation of beta amyloid in sporadic Alzheimer's disease. Med Sci. 2018.

6. Volloch V, Olsen B, Rits S. Precursor-independent overproduction of beta-amyloid in AD: Mitochondrial dysfunction as possible initiator of asymmetric RNA-dependent $\beta$ APP mRNA amplification. An engine that drives Alzheimer's disease. Ann Integr Mol Med. 2019;1(1):1005. http://dx.doi.org/10.33597/aimm.01-1005

7. Volloch V, Olsen B, Rits S. AD "Statin": Alzheimer's disorder is a "fast" disease preventable by therapeutic intervention initiated even late in life and reversible at the early stages. Ann Integr Mol Med. 2020;2(1):1006. http://dx.doi.org/10.33597/aimm.02-1006

8. Volloch V, Olsen B, Rits S. Alzheimer's disease is driven by intraneuronally retained beta-amyloid produced in the $\mathrm{AD}$-specific, $\beta \mathrm{APP}$-independent pathway: Current perspective and experimental models for tomorrow. Ann Integr Mol Med. 2020;2(1):1007. http://dx.doi.org/10.33597/aimm.02-1007

9. Egan M, Kost J, Tariot P. Randomized trials of verubecestat for mild-to-moderate Alzheimer's disease. N Engl J Med. 2018;378:1691-1703.

10. Volloch V, Schweitzer B, Rits S. Antisense globin RNA in murine erythroid tissues: Structure, origin and possible function. Proc. Natl. Acad. Sci. USA. 1996,93: 24762481.

11. Volloch V. Protein-encoding RNA to RNA information transfer in mammalian cells: Principles of RNA-dependent mRNA amplification. Ann Integr Mol Med. 2019;1(1):1002. http://dx.doi.org/10.33597/aimm.01-1002

12. Rits S, Olsen B, Volloch V. Protein-encoding RNA to RNA information transfer in mammalian cells: RNA-dependent mRNA amplification. Identification of 
chimeric RNA intermediates and putative RNA end products. Ann Integr Mol Med. 2019;1(1):1003. http://dx.doi.org/10.33597/aimm.01-1003

13. Volloch V, Rits S, Olsen B. RNA-dependent amplification of mammalian mRNA encoding extracellullar matrix proteins: Identification of chimeric RNA intermediates for $\alpha 1, \beta 1$, and $\gamma 1$ chains of laminin. Ann Integr Mol Med. 2019;1(1):1004 http://dx.doi.org/10.33597/aimm.01-1004

14. Kapranov P, Ozsolak F, Kim S, Foissac S, Lipson D, Hart C, et al. New class of genetermini-associated human RNAs suggests a novel RNA copying mechanism. Nature. 2010;466:642-6

15. Lehmann E, Brueckner F, Cramer P. Molecular basis of RNA-dependent RNA polymerase II activity. Nature. 2007;450:445-9.

16. Wagner S, Yakovchuk P, Ponicsan S, Drullinger L, Kugel J, Goodrich J. RNA polymerase II acts as an RNA-dependent RNA polymerase to extend and destabilize non-coding RNA. EMBO J. 2013;32:781-90.

17. Maida Y, Yasukawa M, Masutomi K. De Novo RNA Synthesis by RNA-Dependent RNA Polymerase Activity of Telomerase Reverse Transcriptase. Mol Cell Biol. 2016;38:1248-59.

18. Volloch V, Schweitzer B, Rits S. Evolutionarily conserved elements in the 5 -untranslated region of $\beta$ eta-globin mRNA mediate site-specific priming of a unique hairpin structure during cDNA synthesis. Nucl Acids Res. 1994;22:5302-9.

19. Pakos-Zebrucka K, Koryga I, Mnich K. Integrated stress response. EMBO Rep 2016;17:1374-95.

20. Quiros P, Pedro M, Zamboni M. Multi-omics analysis identifies ATF4 as a key regulator of the mitochondrial stress response in mammals. J Cell Biol. 2017;216:2027-45.

21. Breimer L, Denny P. Alzheimer amyloid aspects. Nature. 1987;326:749-50.

22. Dyrks T, Dyrks E, Monning U, Urmoneit B, Turner J, Beyreuther K. Generation of beta A4 from the amyloid protein precursor and fragments thereof. FEBS Let. 1993;335:89-93.

23. Haass C, Lemere C, Capell A, Citron M, Seubert P, Schenk D, et al. The Swedish mutation causes early-onset Alzheimer's disease by beta-secretase cleavage within the secretory pathway. Nat Med. 1995;1:1291-6.

24. Iizuka T, Shoji M, Kawarabayashi T, Sato M, Kobayashi T. Intracellular generation of amyloid beta-protein from amyloid beta-protein precursor fragment by direct cleavage with beta- and gamma-secretase. Biochem. Biophys. Res Commun. 1996;218:238-42.

25. Citron M, Haass C, Selkoe D. Production of amyloid beta peptide by cultured cells: no evidence for internal initiation of translation at Met596. Neurobiol Aging. 1993;14:571-3.

26. Mita S, Sadlock J, Herbert J, Schon E. A cDNA specifying the human amyloid beta precursor protein encodes a 95-kDa polypeptide. Nucl Acids Res. 1988;16:9351.

27. Mita S, Sadlock J, Herbert J, Schon E. A cDNA specifying the human amyloid beta precursor protein encodes a $95-\mathrm{kDa}$ polypeptide: Correction. Nucl Acids Res. 1988;16:11402.

28. Salbaum J, Weidemann A, Lemaire H, Masters C, Beyreuther K. The promoter of Alzheimer's disease amyloid A4 precursor gene. EMBO J. 1988;7:2807-13.

29. Chu Q, Martinez T, Novak S, Donaldson C, Tan D. Regulation of the ER stress response by a mitochondrial microprotein. Nature Commun. 2019;10:4883.

30. Yoshida H, Matsui T, Yamamoto A, Okada T, Mori K. XBP1 mRNA is induced by ATF6 and spliced by IRE1 in response to ER stress to produce a highly active transcription factor. Cell. 2001;107(7):881-91.

31. Jiang S, Zhang E, Zhang R, Li X. Altered activity patterns of transcription factors induced by endoplasmic reticulum stress. BMC Biochem. 2016;17:8.

32. Fessler E, Eckl E, Shmitt S. A pathway coordinated by DELE1 relays mitochondrial stress to the cytosol. Nature. 2020;579:433-7.

33. Guo X, Aviles G, Tian R. Mitochondrial stress is relayed to the cytosol by an OMA1DELE1-HRI pathway. Nature. 2020;579:427-32.
34. Chafekar S, Baas F, Scheper W. Oligomer-specific amyloid-beta toxicity in cell models is mediated by selective uptake. Biochem Biophys Acta. 2008;9:523-31.

35. Wesen E, Jeffries G, Dzebo M, Esbjorner M. Endocytic uptake of monomeric amyloid- $\beta$ peptides is clathrin- and dynamin-independent and results in selective accumulation of $A \beta(1-42)$ compared to $A \beta(1-40)$. Sci Rep. 2017;7:2021.

36. Hu X, Crick S. Amyloid seeds formed by cellular uptake, concentration, and aggregation of the amyloid-beta peptide. Proc Natl Acad Sci USA. 2009;106:20324-9.

37. Yajima R, Tokutake T, Koyama A. ApoE-isoform-dependent cellular uptake of amyloid- $\beta$ is mediated by lipoprotein receptor LR11/SorLA. Biochem Biophys Res Comm. 2015;456:482-8

38. Omtri R, Davidson M, Arumugam B. Differences in the cellular uptake and intracellular itineraries of amyloid beta proteins 40 and 42: ramifications for the Alzheimer's drug discovery. Mol Pharmaceutics. 2012;9:1887.

39. Bu G, Cam J, Zerbinatti C. LRP in amyloid- $\beta$ production and metabolism. Ann NY Acad Sci. 2006;1086:3553.

40. Wang H. $\beta$-Amyloid ${ }_{1-42}$ binds to $\alpha 7$ nicotinic acetylcholine receptor with high affinity. Implications for Alzheimer's disease pathology. J Biol Chem. 2000;275:562632 .

41. Nagele R, D’Andrea, M, Anderson, W, Wang H. Intracellular accumulation of A $\beta 42$ in neurons is facilitated by the $\alpha 7$ nicotinic acetylcholine receptor in Alzheimer's disease. Neurosci. 2002;110:199-211.

42. Oddo S. Chronic nicotine administration exacerbates tau pathology in a transgenic model of Alzheimer's disease. Proc. Natl Acad Sci USA 2005;102:3046-51.

43. Yan S. RAGE and amyloid- $\beta$ peptide neurotoxicity in Alzheimer's disease. Nature. 1996;382:685-91.

44. Sasaki N. Immunohistochemical distribution of the receptor for advanced glycation end products in neurons and astrocytes in Alzheimer's disease. Brain Res. 2001;888:256-62.

45. Deane R. RAGE mediates amyloid- $\beta$ peptide transport across the blood-brain barrier and accumulation in brain. Nature Med. 2003;9:907-13.

46. Iribarren P, Zhou Y, Hu J, Le Y, Wang, J. Role of formyl peptide receptor-like 1 (FPRL1/FPR2) in mononuclear phagocyte responses in Alzheimer disease. Immunol Res. 2005;31:165-76.

47. Snyder E. Regulation of NMDA receptor trafficking by amyloid- $\beta$. Nature Neurosci. 2005;8:1051-8.

48. LaFerla F, Green K, Oddo S. Intracellular amyloid-beta in Alzheimer's disease. Nat Rev Neurosci. 2007;8(7):499-509.

49. Kinoshita A. Demonstration by FRET of BACE interaction with the amyloid precursor protein at the cell surface and in early endosomes. J Cell Sci. 2003;116:3339-46.

50. Xu H, Greengard P, Gandy S. Regulated formation of Golgi secretory vesicles containing Alzheimer $\beta$ - amyloid precursor protein. J Biol Chem. 1995;270:23243-5.

51. Mizuguchi M, Ikeda K, Kim S. Differential distribution of cellular forms of $\beta$-amyloid precursor protein in murine glial cell cultures. Brain Res. 1992;584:219-25.

52. Cook D. Alzheimer's $A \beta(1-42)$ is generated in the endoplasmic reticulum/ intermediate compartment of NT2N cells. Nature Med. 1997;3:1021-3.

53. Hartmann T. Distinct sites of intracellular production for Alzheimer's disease A $340 / 42$ amyloid peptides. Nature Med. 1997;3:1016-20.

54. Wild-Bode C. Intracellular generation and accumulation of amyloid beta-peptide terminating at amino acid 42. J Biol Chem. 1997;272:16085-8.

55. Lee $\mathrm{S}$. A detergent-insoluble membrane compartment contains $A \beta$ in vivo. Nature Med. 1998;4:730-4.

56. Skovronsky D, Doms R, Lee V. Detection of a novel intraneuronal pool of insoluble amyloid $\beta$ protein that accumulates with time in culture. J Cell Biol. 1998;141:1031-9.

57. Manczak M. Mitochondria are a direct site of $A \beta$ accumulation in Alzheimer's disease neurons: implications for free radical generation and oxidative damage in disease progression. Hum Mol Genet. 2006;15:1437-49. 
58. Sannerud R. Restricted Location of PSEN2/gamma-secretase determines substrate specificity and generates an intracellular Abeta pool. Cell1. 2016;66:193-208.

59. Wesen E, Jeffries G, Dzebo M, Esbjorner M. Endocytic uptake of monomeric amyloid- $\beta$ peptides is clathrin- and dynamin-independent and results in selective accumulation of $A \beta(1-42)$ compared to $A \beta(1-40)$. Sci Rep. 2017;7:2021.

60. Hansson C. Nicastrin, presenilin, APH-1, and PEN-2 form active ?-secretase complexes in mitochondria. J Biol Chem. 2004;279: 51654-60.

61. Caspersen C. Mitochondrial A $\beta$ : a potential focal point for neuronal metabolic dysfunction in Alzheimer's disease. FASEB J. 2005;19:2040-1.

62. Keskin A, Kekuš M, Adelsberger H, Neumann U, Shimshek D. BACE inhibitiondependent repair of Alzheimer's pathophysiology. Proc Natl Acad Sci USA. 2017;114:8631-6.
63. Hu X, Das B, Hou H, He W, Yan R. BACE1 deletion in the adult mouse reverses preformed amyloid deposition and improves cognitive functions. J Exp Med. 2018;10:1084.

64. Teo E, Ravi S, Barardo D, Kim H, Fong S. Metabolic stress is a primary pathogenic event in transgenic Caenorhabditis elegans expressing pan-neuronal human amyloid beta. eLife. 2019

65. Choi S. A three-dimensional human neural cell culture model of Alzheimer's disease. Nature. 2014;515:274-8 